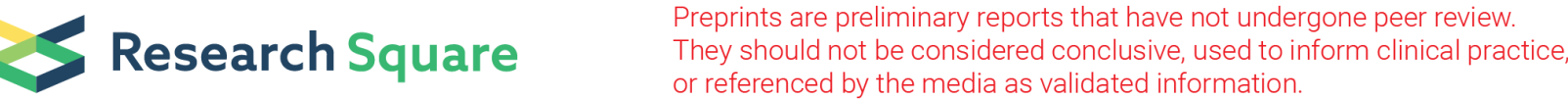

\section{E2F2 inhibition induces autophagy to impair metastasis via the PI3K/Akt/mTOR pathway in gastric cancer}

Hui Li

Qingdao University

Shufen Zhao

The Affiliated Hospital of Qingdao University

Liwei Shen

Qingdao Women and Children's Hospital

Peige Wang

The Affiliated Hospital of Qingdao University

Shihai Liu

The Affiliated Hospital of Qingdao University

Yingji Ma

Qingdao University

Zhiwei Liang

Qingdao University

Gongjun Wang

Qingdao University

Jing Lv

The Affiliated Hospital of Qingdao University

Wensheng Qiu ( $\square$ wsqiuqd@163.com )

Affiliated Hospital of Qingdao University

Research

Keywords: gastric cancer, E2F2, autophagy, metastasis, PI3K/Akt/mTOR pathway

Posted Date: October 29th, 2020

DOI: https://doi.org/10.21203/rs.3.rs-97865/v1

License: (c) (1) This work is licensed under a Creative Commons Attribution 4.0 International License.

Read Full License 


\section{Abstract}

Background: E2F2 is a member of the E2F family of transcription factors with important yet incompletely understood biological functions in cancer. In some cancer types, controversial tumor-promoting and tumor-suppressive roles of E2F2 have been reported. However, the biological role of E2F2 in gastric cancer (GC) remains to be determined.

Methods: We analyzed E2F2 expression via multiple gene expression databases. The prognostic value of the E2F2 was determined by Kaplan-Meier Plotter and Cox regression. The correlations between E2F2 and cancer immune infiltrates were investigated via Tumor Immune Estimation Resource (TIMER). The functions and pathways of E2F2 and its 50 frequently changed genes closely associated with the family members were analyzed using Database for Annotation, Visualization, and Integrated Discovery (DAVID) software. We used immunohistochemistry (IHC), quantitative real-time PCR (qPCR) and western blot to verify the expression level of E2F2 in GC and further studied the effects of E2F2 on PI3K/Akt/mTOR activity; GC cell autophagy, migration, and invasion through wound healing assays, transwell assays, Western blotting, and transmission electron microscopy.

Results: We observed that compared with normal gastric tissues/cells, E2F2 is highly expressed in gastric cancer tissues and cells in both the public datasets and in our experimental verification. High E2F2 expression was associated with poorer overall survival (OS). Moreover, E2F2 expression showed strong correlations with diverse immune marker sets in GC. Moreover, E2F2 overexpression promoted GC cell migration and invasion in vitro by inactivating PI3K/Akt/mTOR-mediated autophagy. Conversely, E2F2 inhibition suppressed GC cell migration and invasion in vitro by activating PI3K/Akt/mTOR-mediated autophagy.

Conclusions: In conclusion, this study provides multi-level evidence for the importance of E2F2 in gastric carcinogenesis and its potential as a biomarker in GC. We demonstrated that E2F2 is overexpressed in GC and that high E2F2 expression is associated with aggressive tumor features and poorer patient prognosis. Further, our results suggest a potential novel immune regulatory role of E2F2 in tumor immunity. Functionally, we discovered a new role of E2F2 in regulating PI3K/Akt/mTOR-mediated autophagy and the downstream processes of cell migration and invasion. Our results could potentially reveal new targets and strategies for GC diagnosis and treatment.

\section{Background:}

Gastric cancer (GC) is the fifth most common malignancy in the world and the third leading cause of cancer-related death (1). Due to the high rate of recurrence and metastasis, the five-year survival rate for advanced GC is poor (2). However, existing targeted drugs show unsatisfactory efficacy, due to a combination of factors spanning an array of different clinical and biological behaviors, and the development of anti-GC drug resistance (3). The molecular mechanisms underlying tumor formation and progression are poorly understood, which further complicates the effective treatment of GC (4). In 
addition, the lack of markers that are specific for tumor type or disease stage represents a critical gap in the current understanding and treatment of GC.

E2Fs is a family of transcription factor proteins that are considered to be the main regulators of cell growth and proliferation. The main functions of E2F proteins are to regulate the cell cycle, cell differentiation, the DNA damage response and cell death (5). Whereas certain members of E2Fs, such as E2F1, has been extensively studied (6-9), the biological effects of E2F2 in GC remain largely unknown. Previous studies have shown that E2F2 plays contradictory roles in the development of tumors. On the one hand, E2F2 can inhibit tumorigenesis by inhibiting cell cycle regulators (10). On the other hand, E2F2 can act as an "activator" to increase target expression and cause cancer (11). In gastric cancer, whether E2F2 exists as an oncogene or a tumor suppressor gene is worthy of our further research. This study aims to fill in the gaps in understanding the contribution of E2F2 to GC development and/or progression, which may form the basis for developing new therapeutic strategies for GC.

Tumor metastasis causes $>90 \%$ of cancer deaths, and there is increasing evidence that autophagy is intricately involved in tumor metastasis $(12,13)$. Autophagy is a highly conserved catabolic process, which maintains cellular homeostasis by targeting damaged proteins or organelles to lysosomal compartments for degradation $(14,15)$; this process is important in both normal human physiology and diseases, including cancer (16). In GC, the inhibition of autophagy was associated with enhanced cell proliferation, migration, and invasion, likely involving activation of the phosphoinositide-3 kinase (PI3K)/Akt/mammalian target of rapamycin (mTOR) signaling pathway (17). While autophagy and the $\mathrm{PI} 3 \mathrm{~K} / \mathrm{Akt} / \mathrm{mTOR}$ pathway appear to play critical roles in GC metastasis, it is unknown whether E2F2 may be a mediator in these processes.

Here, we investigated E2F2 expression and mutations in data from patients with GC in The Cancer Genome Atlas (TCGA) and various public databases. Using multi-dimensional analysis, we evaluated genomic alterations and functional networks related to E2F2 in GC and explored its role in tumor immunity. Finally, we used GC cell lines to verify the high expression of E2F2 in gastric cancer, and examined the functional role(s) of E2F2 in GC cell invasion, migration and autophagy. Our data highlight the novel role of E2F2 in mediating autophagy via the PI3K/Akt/mTOR pathway, with resulting effects on $\mathrm{GC}$ cell metastasis.

\section{Materials And Methods}

\section{ONCOMINE database}

The ONCOMINE database (www.oncomine.org) is an integrated online cancer microarray database containing data from DNA- and RNA-sequencing (DNA-seq and RNA-seq, respectively) analyses used to classify the differential expression between common cancer types and the corresponding normal tissues, as well as clinical and pathological data (18). In our study, the transcriptional expression data for E2F2 in different cancer tissue samples and their corresponding adjacent normal samples were obtained from the 
ONCOMINE database. Differences in transcriptional expression were compared by Student's t test. The pvalue cutoff and fold change threshold were as follows: p-value, 0.01; fold change, 1.5; gene grade, 10\%; and data type, mRNA.

\section{UALCAN database}

UALCAN (http://ualcan.path.uab.edu) is an interactive web resource developed based on the grade 3 RNA sequences and clinical data of 31 cancer types in TCGA database (19). In this study, UALCAN was used to analyze the mRNA expression of E2F2 in primary gastric cancer tissue samples and the relationships of these members with clinicopathological parameters. Differences in transcriptional expression were compared by Student's t test $(p<0.01)$.

\section{GEPIA database}

The Gene Expression Profiling Interactive Analysis (GEPIA) database (http://gepia.cancer-pku.cn/) is an interactive web that includes 9,736 tumors and 8,587 normal samples from TCGA and the GTEx projects (20). In this study, GEPIA was applied to verify the E2F2 expression in GC.

\section{LinkedOmics Database}

The LinkedOmics database (http://www.linkedomics.org/login.php) is a web-based platform for analyzing 32 TCGA cancer-associated multi-dimensional datasets (21). E2F2 co-expression was analyzed statistically using Pearson's correlation coefficient, presenting in volcano plots, heat maps, or scatter plots. The rank criterion was FDR $<0.05$ and 1000 simulations were performed.

\section{5. cBioPortal Database}

Using cBioPortal (22) (https://www.cbioportal.org), the gastric cancer (TCGA, Provisional) dataset (including data from 478 pathological reports) was selected for further E2F2 analysis. The genome map included mutations, copy number changes from GISTIC (CNA), mRNA expression z-scores (RNA-seq V2 RSEM) and protein expression z-scores (RPPA). Mutation, copy number variation (CNV), and gene cooccurrence of E2F2 in GC were analyzed using the c-BioPortal tool.

\section{TIMER database}

TIMER is a comprehensive resource for systematic analysis of immune infiltrates across diverse cancer types from TCGA (https://cistrome.shinyapps.io/timer/), which includes 10,897 samples across 32 cancer types (23). TIMER applies a deconvolution method (24) to infer the abundance of tumorinfiltrating immune cells (TIICs) from gene expression profiles. We analyzed E2F2 expression in STAD and the correlation of E2F2 expression with the abundance of immune infiltrates.

\section{Protein-protein interaction (PPI) network construction and gene enrichment analyses}


The STRING database (http://string-db.org/) provides information regarding the significant associations of PPIs (25). In this study, the STRING database was used to analyze E2F2 and 50 frequently changed genes closely associated with the family members. We used the Database for Annotation, Visualization, and Integrated Discovery (DAVID) (http://www.DAVID.org) (26) to conduct agonistic gene ontology (GO) and Kyoto Encyclopedia of Genes and Genomes (KEGG) analyses of 51 genes, including E2F2 enrichment analysis. $\mathrm{GO}$ enrichment analysis can predict gene function based on biological processes (BPs), cell composition (CC) and molecular function (MF), and KEGG can be used to analyze gene enrichment pathways.

\section{Tissue microarrays (TMAs)}

We collected 60 fresh GC tissues and adjacent tissues for IHC staining and evaluation. The staining intensity was given a score from 0 to 3 based on the following criteria: 0 , no staining; 1 , weak staining; 2 , moderate staining; or 3 , strong staining. All individual patients understood the objectives of this study and provided written informed consent. The Medical Ethics Committee of Qingdao University and the Affiliated Hospital of Qingdao University approved the collection of clinical materials for research purposes.

\section{Cells and culture conditions}

AGS and HGC27 cell lines were purchased from the cell bank of the Chinese Academy of Sciences and were cultured in RPMI-1640 medium containing 10\% fetal bovine serum (FBS) (Gibco, NY, USA). The cells were placed in an incubator at $37^{\circ} \mathrm{C}$ with a $\mathrm{CO} 2$ concentration of $5 \%$.

\section{Transfection}

Cells were transfected with plasmids expressing E2F2 (GV141-E2F2, Genechem, Shanghai, China), plasmids expressing empty vector (GV141-Vector), small interfering RNAs (siRNAs) against E2F2 (siE2F2; GenePharma, Shanghai, China, Supplementary Table 1) and negative control (siNC) using Lipofectamine 2000 (Invitrogen, Carlsbad, CA) according to the manufacturer's instructions.

\section{RNA extraction and qPCR}

Total RNA from cultured cells and frozen tissues was extracted with TRIzol (Invitrogen, Carlsbad, CA). cDNA synthesis was performed using the PrimeScript ${ }^{\text {TM }}$ RT Kit (TaKaRa, Otsu, Japan). SYBR Premix EX Taq $^{\text {TM }}$ (TaKaRa, Otsu, Japan) was used for qPCR on an FTC-3000p Real-Time PCR system (Funglyn Biotech, Shanghai, China). Relative gene expression was determined by the comparative 2- $\Delta \Delta C T$ method. The PCR primers used are listed in Supplementary Table 1.

\section{Western blotting analysis}

Western blotting analysis of protein expression was performed as described previously (27). Briefly, protein lysates $(20 \mu \mathrm{g})$ were separated using sodium dodecyl sulfate-polyacrylamide gel electrophoresis, 
and target proteins were detected by western blotting with antibodies against E2F2 (1:1000) and GAPDH $(1: 50,000)$. Other antibodies used in this study are listed in Supplementary Table 1.

\section{Electron microscopy}

Cells were fixed with $2.5 \%$ glutaraldehyde (Solarbio, Beijing, China) for $4 \mathrm{~h}$ at $4{ }^{\circ} \mathrm{C}$, washed with phosphate-buffered saline (PBS), and post-fixed with $1 \%$ OsO4 buffer for $2 \mathrm{~h}$ at $4{ }^{\circ} \mathrm{C}$. The cells were then washed and dehydrated in a graded series of ethanol solutions and embedded in Epon812 epoxy resin. Ultrathin $(90 \mathrm{~nm})$ sections were collected on copper grids, double-stained with $1 \%$ uranyl acetate and $0.2 \%$ lead citrate, and examined by a JEOL-1200EX transmission electron microscope (Beijing, China).

\section{Cell migration and invasion assays}

Cell migration and invasion were assessed using 8- $\mu$ m-pore transwell compartments (Corning, NY). For migration assays, $5 \times 10^{4}$ cells were suspended in serum-free medium in the upper compartment. After cells were incubated at $37^{\circ} \mathrm{C}$ for $24 \mathrm{~h}$, the translocated cells were stained with $0.5 \%$ crystal violet for 20 min at room temperature. For invasion assays, Matrigel (BD Biosciences, San Jose, CA) was added to each well according to the manufacturer's instructions before $2 \times 10^{5}$ cells were suspended in the upper compartment. After cells were incubated at $37^{\circ} \mathrm{C}$ for $24 \mathrm{~h}$, the translocated cells were incubated with $0.5 \%$ crystal violet for $20 \mathrm{~min}$ at room temperature. For quantification, cells were counted under a light microscope (Nikon, Tokyo, Japan) in five fields (upper, lower, middle, left, right; at $\times 40$ magnification).

\section{Wound-healing assay}

Cell migration was also assessed using the wound-healing assay. Briefly, a wound was generated in a 6well plate by scratching the surface with a $200 \mu \mathrm{L}$ pipette tip. The wounded areas were photographed under a light microscope (Nikon, Tokyo, Japan) at the time the wound was created $(0 \mathrm{~h})$ and at $24 \mathrm{~h}$ after. The percentage of wound healing was calculated using the following formula: [1 - (empty area 24 h/empty area $0 \mathrm{~h})] \times 100$.

\section{Statistical analysis}

All statistical analyses were carried out using the Graphpad Prism 7.0 Software (GraphPad, La Jolla, CA). Categorical data were analyzed using chi-square $(\chi 2)$ test. Cox proportional hazard model was used for univariate and multivariate survival analysis. Survival curves were plotted using the Kaplan-Meier method and compared using log-rank test. Analysis of variance and Student's t test were used for comparison among groups. $\mathrm{P}<0.05$ was considered statistically significant.

\section{Results:}

\section{Elevated expression of E2F2 in GC}


We first evaluated the expression of E2F2 in multiple cancer types. After analyzing more than a dozen cancers including gastric cancer and their corresponding normal tissues, it was found that the mRNA expression of E2F2 in gastric cancer tissues was significantly higher than that of adjacent normal tissues (Figure 1A). Subsequently, we used Oncomine and GEPIA tools to analyze the expression of E2F2 in gastric cancer, and the results showed that compared with normal gastric tissue, the expression level of E2F2 in gastric cancer was significantly increased (Figure B and C). K-M Plotter is used to analyze the effect of E2F2 on OS in patients with gastric cancer. We found that high expression of E2F2 is associated with poor prognosis of patients (Figure 1D). Further sub-group analysis of multiple clinic-pathological features of TCGA-STAD samples in UALCAN database consistently showed elevated transcription level of E2F2. The expression of E2F2 was significantly higher in GC patients than normal controls in subgroup analysis based on gender, age, ethnicity, disease stages, and tumor grade (Figure 2). Thus, E2F2 expression may serve as a potential diagnostic indicator in GC.

\section{Independent prognostic value of E2F mRNA expression in terms of OS in gastric cancer patients}

we downloaded the clinical and E2F mRNA expression data of 407 gastric cancer patients from the TCGA database (Table 1). We used survival, glmnet and other software packages to integrate and standardize the downloaded raw data and then performed univariate and multivariate Cox survival regression analyses. In the univariate analysis, we found that age, regional lymph node involvement, distant metastasis, pathological stage, and E2F2 are closely related to patient survival (Table 2). In the multivariate analysis, we found that a higher pathological stage $(p=0.036)$ and higher E2F2 mRNA expression $(p=0.005)$ were related to shorter OS of gastric cancer patients (Table 3 ).

\section{Co-expression analysis and Genomic alterations of E2F2 in GC}

To gain the insight of E2F2 biological meaning in GC, the function module of LinkedOmics was used to examine E2F2 co-expression mode in STAD cohort. As shown in Figure 3A, 9963 genes (dark red dots) were shown significant positive correlations with E2F2, whereas 10262 genes (dark green dots) were shown significant negative correlations (false discovery rate, FDR $<0.01$ ). The top 50 significant genes positively and negatively correlated with E2F2 were shown in the heat map (Figure 3B). We then used the cBioPortal tool to determine the types and frequency of E2F2 alterations in GC based on DNA sequencing data from STAD patients. E2F2 was altered in 16 of 473 (3\%) STAD patients (Figure 3C). These alterations include Missense Mutation in 5 cases, mRNA High in 5 cases, Amplification in 4 cases and Deep Deletion in 1cases. As shown in Figure 3D, compared with the diploid group, the E2F2 expression level in the gain or amplification group was higher. Next, the frequency distribution of E2F2 CNV patients in different stage and grade groups was presented in Figure $3 \mathrm{E}$ and $3 \mathrm{~F}$, suggesting the high occurrence and an early-event of E2F2 CNV alteration in GC.

\section{Relationships of E2F2 with immune cells and PD-1/PD-L1}

Search the TIMER database to estimate the correlation between E2F2 mRNA expression and immune cell infiltration. As shown in Figure 4A, after adjusting the purity, the expression of E2F2 in GC is compared 
with M1 cells (Figure 4A), M2 cells (Figure 4B), Th1 cells (Figure 4C), Th2 cells (Figure 4D) and Treg cells (Figure 4E) Significant correlation. Moreover, due to the promising prospect of immunotherapy, we further determined the association between E2F2 expression and PD-1 and PD-L1 (Figure 4F). We also noticed a positive correlation of E2F2 with PD-1 $(r=0.185, P=1.49 \mathrm{e}-4)$ and PD-L1 $(r=0.279, P=8.67 \mathrm{e}-9)$. Using the data of GISTIC2.0, the effect of different copy status of E2F2 on immune infiltration compared with normal tissues was tested. We observed that E2F2 CNV has a significant correlation with the infiltration level of $B$ cells, CD8+ T cells $\square C D 4+T$ cells, macrophages, neutrophils and dendritic cells (Figure 4G).

\section{Protein-protein interaction (PPI) network construction and gene enrichment analyses}

In order to understand the function of E2F2 in gastric cancer, we used the STRING website to explore the proteins that can interact with E2F2, selected the top 50 genes with high correlation, and used the cytoscape tool to construct an integrated network (Figure $5 \mathrm{~A}$ ). We found that genes related to the cell cycle, including CCNE1, CCNE2, CDK2, CDK4, CDKN1 and CDKN2A, are closely related to the changes in E2F2. Figure 5B showed 10 hub genes closely related to E2F2. Next, The functions of E2F2 and its 50 neighboring genes were analyzed by GO and KEGG analyses in DAVID. GO enrichment analysis predicts gene function from three aspects, namely, BPs, CCs and MFs. As shown in Figure $5 \mathrm{C}$, we found that in BPs, target genes were mainly enriched in biological regulation $\llbracket$ metabolic process and response to stimulus; in CCs, target genes were enriched in membrance,nucleus and membrance-enclosed lumen; and the MFs were mainly protein binding,ion binding and nucleic acid binding. KEGG enrichment analysis (Figure 4D) shows that these genes are most enriched in cell cycle related pathways, which is consistent with the PPI analysis results. It is worth noting that PI3K-Akt signaling pathway also showed a high degree of enrichment, which attracted our attention.

\section{E2F2 is a regulator of the PI3K/Akt/mTOR pathway}

Therefore, we want to further verify the relationship between E2F2 and PI3K-Akt signaling pathway through in vitro experiments. To verify the high expression of E2F2 in GC, we subjected $60 \mathrm{GC}$ tissues and their adjacent tissues to immunohistochemical staining $(\mathrm{IHC})$. Representative images are shown in Figure $6 \mathrm{~A}$. The level of E2F2 expression in normal tissues adjacent to cancer tissues was significantly lower than that in the cancer tissues. We used GPCR and western blotting of the matched GC and adjacent nontumor frozen tissues and similarly observed significant overexpression of E2F2 in GC (Figure 6B and 6C). We use PCR and Western blot experiments to explore the expression of E2F2 in GC cells. We found that the mRNA and protein expression levels of E2F2 were significantly increased in AGS and HGC27 compared to GES-1 cells (Figure 6D and 6E). In the next experiment, we transfected HGC27 cells and AGS cells with GV141-E2F2 and siE2F2, respectively, to better understand the effect of E2F2 on the biological behavior of gastric cancer. High overexpression efficiency and knockdown efficiency were observed in the treated cells (Figure 6F and G). Subsequently, we evaluated the effect of E2F2 on the PI3K/Akt/mTOR pathway in AGS cells. Western blotting showed that the expression levels of PI3K 110 Akt), and phospho-mTOR (p-mTOR) levels increased in HGC27 cells at $24 \mathrm{~h}$ after E2F2 overexpression 
(Figure $6 \mathrm{H}$ ). Conversely, following E2F2 knockdown with siE2F2 for $24 \mathrm{~h}$, the expression levels of PI3K $110 \beta, \mathrm{p}-\mathrm{Akt}$, and p-mTOR were markedly decreased in AGS cells (Figure 6I).

\section{Effects of E2F2 expression levels on GC cell migration and invasion via autophagy mediation}

Since E2F2 can regulate the PI3K/Akt/mTOR pathway, which play an important role in affecting autophagy $(28,29)$, we further examined the relationship between E2F2 and autophagy-related protein expression in GC cells that were transfected with GV141-EE2F2 or siE2F2. We found that the expression levels of LC3II decreased most markedly after E2F2 was overexpressed, while P62 protein expression was significantly increased (Figure 7A). Conversely, the expression levels of LC3II increased most markedly after E2F2 was inhibited, while P62 protein expression was significantly reduced (Figure 7B). Interestingly, the number of autophagic vesicles was also significantly decreased in HGC27 cells transfected with GV141-E2F2, and the number of autophagic vesicles was observed to be greatest in AGS cells transfected with siE2F2 (Figure 7C). Collectively, our data demonstrated that E2F2 overexpression inhibited autophagy and E2F2 inhibition induced autophagy in GC cells. We observed E2F2 overexpression significantly increased cell migration and invasion (Figure7D and 7E) in GC cells. Moreover, E2F2 suppression-mediated anti-metastasis effects were reversed when cells were co-treated with siE2F2 and the autophagy inhibitor 3-MA (Figure 7F and 7G). We also confirmed that co-treatment with 3-MA significantly decreased E2F2 inhibition-dependent LC3-II and Beclin1 protein expression and increasd P62 and MMP9 protein expression(Figure 7H). Thus our results demonstrated that E2F2 overexpression promoted GC cell migration and invasion via autophagy inhibition. However, E2F2 suppression induced autophagy, resulting in inhibition of GC cell migration and invasion.

\section{Discussion:}

E2F2 plays contradictory roles in the development of tumors. On the one hand, E2F2 can inhibit tumorigenesis by inhibiting cell cycle regulators. On the other hand, E2F2 can act as an "activator" to increase target expression and cause cancer (30). Previous studies have shown that changes in E2F2 protein expression are closely related to the occurrence of different cancers. It has been reported that knocking down E2F2 significantly reduces the metastatic ability of breast cancer cells, and mutations in E2F2 are related to tumor proliferation and survival in breast cancer patients (11). E2F2 functions as an oncogene in liver cancer (10), while in prostate cancer, E2F2 inhibits tumor cell proliferation by targeting miRNAs (31). However, The role of E2F2 in gastric cancer has not been fully confirmed. To gain more detailed insights into the potential functions of E2F2 in GC and its regulatory network, We conducted bioinformatics analysis of public data and experimental verification to guide the future research of gastric cancer.

Using large independent datasets, we confirmed the significantly enhanced expression of E2F2 in GC tissues compared to non-tumor gastric tissues. GC patients with higher E2F2 expression have poorer prognosis than those with lower E2F2 expression; Cox regression models consistently established E2F2 expression level in GC is an independent predictor of the OS of GC patients. Further sub-group analysis of 
multiple clinic-pathological features of TCGA-STAD samples showed that the expression of E2F2 was significantly higher in GC patients than normal controls in subgroup analysis based on gender, age, ethnicity, disease stages, and tumor grade. Thus, our results suggest that E2F2 up-regulation occurs in many cases of GC and deserves further clinical validation as a potential diagnostic and prognostic marker.

A recent study found that genomic alteration, such as somatic mutations in the genes encoding components of the spliceosome, occurs frequently in human neoplasms (32). CNVs can have major genomic implications, such as disrupting genes, altering genetic content, and lead to phenotypic differences (33). Our study found that the copy number of E2F2 was increased in GC and that the major type of E2F2 alteration was Missense Mutation and Amplification, which was associated with shorter survival.

The tumor microenvironment is the non-cancerous cells present in and around a tumor, having a strong influence on the genomic analysis of tumor samples (34).Tumor-infiltrating immune cells are part of the complex microenvironment and are associated with the biological behavior and patient survival in GC (35). In this study, we observed that E2F2 expression was positively correlated with M1/M2 cells, Th1/Th2 cells, Tregs and Macrophages in GC tissues. According to a previous research, E2F2 are differentially required for homeostasis-driven and antigen-induced T cell proliferation in vivo (9).Azkargorta et al. found that E2F2 modulates cellular sensitivity to xenobiotic signals through the negative regulation of the Ahr pathway in T Lymphocytes (36). Our results support the findings that E2F2 is specifically correlated with immune infiltrating cells in GC, which suggests that E2F2 plays a vital role in immune infiltration in the tumor microenvironment. Further studies need to be done to elucidate whether E2F2 is a crucial factor in mediating immunity therapy.

Our observed correlation of E2F2 expression with the mTOR pathway (from KEGG analysis) led us to investigate the novel role of E2F2 in autophagy, a process which is modulated by the PI3K/Akt/mTOR pathway. Specifically, our data demonstrated that E2F2 overexpression upregulated the PI3K/Akt/mTOR pathway, resulting in inhibition of autophagy and promotion of GC cell migration and invasion in vitro. And E2F2 inhibition downregulated the PI3K/Akt/mTOR pathway, resulting in induction of autophagy and inhibition of GC cell migration and invasion. The autophagy inhibitor, 3-MA, partially reversed the effects of E2F2 inhibition on GC cell migration and invasion, further confirming the contribution of autophagy toward these processes. Previous studies have reported that E2F1 can regulate the effect of autophagy (37), and E2F2, as a member of the E2Fs transcription factor family, its role in autophagy has not been confirmed. Our research innovatively discovered that E2F2 may play an important role in autophagy. This may provide new insights into the dual role of E2F2 in cancer cells. It is possible that E2F2 regulates GC growth and progression in a complex manner, perhaps through the modulation of autophagy, which has been shown to be a double-edged sword in cancers, including GC.

\section{Conclusion}


This study provides multi-level evidence for the importance of E2F2 in gastric carcinogenesis and its potential as a biomarker in GC. We demonstrated that E2F2 is overexpressed in GC and that high E2F2 expression is associated with aggressive tumor features and poorer patient prognosis. In addition, our analyses revealed that the levels of immune infiltration and a list of immune markers were significantly correlated with E2F2 expression in GC. Our analyses provide novel insights into the prognostic role of E2F2 and potential role of E2F2 in the tumor immunology of GC. Functionally, we firstly uncovered a novel mechanism of E2F2 in regulating autophagy at least in part via the PI3K/Akt/mTOR pathway, which in turn regulated GC cell migration and invasion. These findings call for large-scale clinical research and deeper mechanism studies. Nevertheless, the current results are encouraging, and noteworthy in the field of identifying promising prognostic biomarkers for GC.

\section{Abbreviations}

GC: gastric cancer; TIMER: Tumor Immune Estimation Resource; DAVID: Database for Annotation, Visualization, and Integrated Discovery; OS: overall survival; PI3K: phosphoinositide-3 kinase; mTOR: mammalian target of rapamycin; TCGA: The Cancer Genome Atlas; GEPIA: Gene Expression Profiling Interactive Analysis; GO: gene ontology ; KEGG: Kyoto Encyclopedia of Genes and Genomes; BPs: biological processes; CC: cell composition ; MF: molecular function; TMAs: Tissue microarrays; IHC: Immunohistochemistry; FBS: fetal bovine serum; qPCR: quantitative real-time PCR; PBS: phosphatebuffered saline; CNV: copy number variation

\section{Declarations}

\section{Ethics approval and consent to participate}

Collection of samples used in this study was approved by the Ethics Committee of The Affiliated Hospital of Qingdao University

\section{Consent for publication}

Not applicable.

\section{Availability of data and materials}

All data generated or analysed during this study are included in this published article. Further details are available from the corresponding author upon reasonable request.

\section{Competing interests}

The authors declare that they have no conflict of interest.

\section{Funding}


This study is funded by Natural Science Foundation of China (81602068), WU JIEPING MEDICAL FOUNDATION(320.6750.19088-29) and Beijing Xisike Clinical Oncology Research Foundation(Y-HR2018185).

\section{Authors' contributions}

$\mathrm{HL}$ and SZ analyzed the data and wrote the manuscript. LS, PW and SL assisted in editing the manuscript. YM, ZL and GW contributed to the design of the study. WQ and JL are the corresponding authors of the paper. All authors read and approved the final manuscript.

\section{Acknowledgements}

I shall extend my thanks to Ms. Tang for all her kindness and help.

\section{References}

1. Bray F, Ferlay J, Soerjomataram I, Siegel RL, Torre LA, Jemal A. Global cancer statistics 2018 : GLOBOCAN estimates of incidence and mortality worldwide for 36 cancers in 185 countries. CA Cancer J Clin. 2018;68(6):394-424.

2. Ang TL, Fock KM. Clinical epidemiology of gastric cancer. Singapore Med J. 2014;55(12):621-8.

3. Yang W, Raufi A, Klempner SJ. Targeted therapy for gastric cancer: molecular pathways and ongoing investigations. Biochim Biophys Acta. 2014;1846(1):232-7.

4. Marques-Lespier JM, Gonzalez-Pons M, Cruz-Correa M. Current Perspectives on Gastric Cancer. Gastroenterol Clin North Am. 2016;45(3):413-28.

5. Gaubatz S, Lindeman GJ, Ishida S, Jakoi L, Nevins JR, Livingston DM, et al. E2F4 and E2F5 play an essential role in pocket protein-mediated G1 control. Mol Cell. 2000;6(3):729-35.

6. Huang CL, Liu D, Nakano J, Yokomise H, Ueno M, Kadota K, et al. E2F1 overexpression correlates with thymidylate synthase and survivin gene expressions and tumor proliferation in non small-cell lung cancer. Clin Cancer Res. 2007;13(23):6938-46.

7. Ladu S, Calvisi DF, Conner EA, Farina M, Factor VM, Thorgeirsson SS. E2F1 inhibits c-Myc-driven apoptosis via PIK3CA/Akt/mTOR and COX-2 in a mouse model of human liver cancer. Gastroenterology. 2008;135(4):1322-32.

8. Xie Y, Yin Y, Li L, Ma Y, Xiao Q. Short interfering RNA directed against the E2F-1 gene suppressing gastric cancer progression in vitro. Oncol Rep. 2009;21(5):1345-53.

9. Yamazaki K, Yajima T, Nagao T, Shinkawa H, Kondo F, Hanami K, et al. Expression of transcription factor E2F-1 in pancreatic ductal carcinoma: an immunohistochemical study. Pathol Res Pract. 2003;199(1):23-8.

10. Dong Q, Meng P, Wang T, Qin W, Qin W, Wang F, et al. MicroRNA let-7a inhibits proliferation of human prostate cancer cells in vitro and in vivo by targeting E2F2 and CCND2. PLoS One. 2010;5(4):e10147. 
11. Bollig-Fischer A, Marchetti L, Mitrea C, Wu J, Kruger A, Manca V, et al. Modeling time-dependent transcription effects of HER2 oncogene and discovery of a role for E2F2 in breast cancer cell-matrix adhesion. Bioinformatics. 2014;30(21):3036-43.

12. Huang F, Wang BR, Wang YG. Role of autophagy in tumorigenesis, metastasis, targeted therapy and drug resistance of hepatocellular carcinoma. World J Gastroenterol. 2018;24(41):4643-51.

13. Wang S, Zhu M, Wang Q, Hou Y, Li L, Weng H, et al. Alpha-fetoprotein inhibits autophagy to promote malignant behaviour in hepatocellular carcinoma cells by activating PI3K/AKT/mTOR signalling. Cell Death Dis. 2018;9(10):1027.

14. Kroemer G, Jaattela M. Lysosomes and autophagy in cell death control. Nat Rev Cancer. 2005;5(11):886-97.

15. Rabinowitz JD, White E. Autophagy and metabolism. Science. 2010;330(6009):1344-8.

16. White E, DiPaola RS. The double-edged sword of autophagy modulation in cancer. Clin Cancer Res. 2009;15(17):5308-16.

17. Cao Y, Luo Y, Zou J, Ouyang J, Cai Z, Zeng X, et al. Autophagy and its role in gastric cancer. Clin Chim Acta. 2019;489:10-20.

18. Rhodes DR, Yu J, Shanker K, Deshpande N, Varambally R, Ghosh D, et al. ONCOMINE: a cancer microarray database and integrated data-mining platform. Neoplasia. 2004;6(1):1-6.

19. Chandrashekar DS, Bashel B, Balasubramanya SAH, Creighton CJ, Ponce-Rodriguez I, Chakravarthi B, et al. UALCAN: A Portal for Facilitating Tumor Subgroup Gene Expression and Survival Analyses. Neoplasia. 2017;19(8):649-58.

20. Tang Z, Li C, Kang B, Gao G, Li C, Zhang Z. GEPIA: a web server for cancer and normal gene expression profiling and interactive analyses. Nucleic Acids Res. 2017;45(W1):W98-W102.

21. Sun CC, Li SJ, Chen ZL, Li G, Zhang Q, Li DJ. Expression and Prognosis Analyses of Runt-Related Transcription Factor Family in Human Leukemia. Mol Ther Oncolytics. 2019;12:103-11.

22. Gao J, Aksoy BA, Dogrusoz U, Dresdner G, Gross B, Sumer SO, et al. Integrative analysis of complex cancer genomics and clinical profiles using the cBioPortal. Sci Signal. 2013;6(269):pl1.

23. Li T, Fan J, Wang B, Traugh N, Chen Q, Liu JS, et al. TIMER: A Web Server for Comprehensive Analysis of Tumor-Infiltrating Immune Cells. Cancer Res. 2017;77(21):e108-e10.

24. Li B, Severson E, Pignon JC, Zhao H, Li T, Novak J, et al. Comprehensive analyses of tumor immunity: implications for cancer immunotherapy. Genome Biol. 2016;17(1):174.

25. Szklarczyk D, Franceschini A, Kuhn M, Simonovic M, Roth A, Minguez P, et al. The STRING database in 2011: functional interaction networks of proteins, globally integrated and scored. Nucleic Acids Res. 2011;39(Database issue):D561-8.

26. Huang da W, Sherman BT, Lempicki RA. Systematic and integrative analysis of large gene lists using DAVID bioinformatics resources. Nat Protoc. 2009;4(1):44-57.

27. Liu W, Wang X, Wang Y, Dai Y, Xie Y, Ping Y, et al. SGK1 inhibition-induced autophagy impairs prostate cancer metastasis by reversing EMT. J Exp Clin Cancer Res. 2018;37(1):73. 
28. Xu Z, Han X, Ou D, Liu T, Li Z, Jiang G, et al. Targeting PI3K/AKT/mTOR-mediated autophagy for tumor therapy. Appl Microbiol Biotechnol. 2020;104(2):575-87.

29. Kim YC, Guan KL. mTOR: a pharmacologic target for autophagy regulation. J Clin Invest. 2015;125(1):25-32.

30. Chong JL, Wenzel PL, Saenz-Robles MT, Nair V, Ferrey A, Hagan JP, et al. E2f1-3 switch from activators in progenitor cells to repressors in differentiating cells. Nature. 2009;462(7275):930-4.

31. Wang H, Zhang X, Liu Y, Ni Z, Lin Y, Duan Z, et al. Downregulated miR-31 level associates with poor prognosis of gastric cancer and its restoration suppresses tumor cell malignant phenotypes by inhibiting E2F2. Oncotarget. 2016;7(24):36577-89.

32. Andor N, Maley CC, Ji HP. Genomic Instability in Cancer: Teetering on the Limit of Tolerance. Cancer Res. 2017;77(9):2179-85.

33. Katoh M. Cancer genomics and genetics of FGFR2 (Review). Int J Oncol. 2008;33(2):233-7.

34. Aran D, Sirota M, Butte AJ. Systematic pan-cancer analysis of tumour purity. Nat Commun. 2015;6:8971.

35. Kwak Y, Seo AN, Lee HE, Lee HS. Tumor immune response and immunotherapy in gastric cancer. J Pathol Transl Med. 2020;54(1):20-33.

36. Azkargorta M, Fullaondo A, Laresgoiti U, Aloria K, Infante A, Arizmendi JM, et al. Differential proteomics analysis reveals a role for E2F2 in the regulation of the Ahr pathway in T lymphocytes. Mol Cell Proteomics. 2010;9(10):2184-94.

37. Polager S, Ofir M, Ginsberg D. E2F1 regulates autophagy and the transcription of autophagy genes. Oncogene. 2008;27(35):4860-4.

\section{Tables}

Table1 Basic characteristics of 366 GC patients. 


\begin{tabular}{|c|c|}
\hline Variables & nts $₫ N=366 \rrbracket$ \\
\hline Gender( Male/female) & $235 / 131$ \\
\hline Age(years, Mean $\pm S D)$ & $65.57 \pm 10.27$ \\
\hline \multicolumn{2}{|l|}{ Tumor } \\
\hline $\mathrm{T} 1$ & 17 \\
\hline $\mathrm{T} 2$ & 81 \\
\hline T3 & 169 \\
\hline T4 & 98 \\
\hline \multicolumn{2}{|l|}{ Regional lymph node } \\
\hline No & 114 \\
\hline N1 & 97 \\
\hline N2 & 78 \\
\hline N3 & 70 \\
\hline \multicolumn{2}{|l|}{ Metastasis } \\
\hline MO & 325 \\
\hline M1 & 24 \\
\hline \multicolumn{2}{|l|}{ Histologic grade } \\
\hline 1 & 8 \\
\hline 2 & 129 \\
\hline 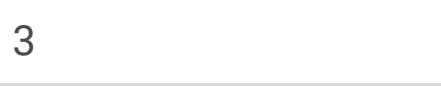 & 220 \\
\hline \multicolumn{2}{|l|}{ Pathologic stage } \\
\hline 1 & 51 \\
\hline 2 & 124 \\
\hline 3 & 151 \\
\hline 4 & 39 \\
\hline
\end{tabular}

Table2 Univariate analysis of overall survival in $366 \mathrm{GC}$ specimens. 


\begin{tabular}{|c|c|c|c|}
\hline \multirow[t]{2}{*}{ Variables } & \multicolumn{3}{|c|}{ Univariate analysis } \\
\hline & Hazard Ratio & $95 \% \mathrm{Cl}$ & $P$ value \\
\hline Gender & 0.775 & $0.544-1.105$ & 0.160 \\
\hline Age(years) & 1.022 & $1.005-1.039$ & 0.011 \\
\hline Tumor & & & 0.109 \\
\hline $\mathrm{T}(1)$ & 0.098 & $0.013-0.710$ & 0.022 \\
\hline $\mathrm{T}(2)$ & 0.768 & $0.480-1.229$ & 0.271 \\
\hline $\mathrm{T}(3)$ & 0.882 & $0.601-1.294$ & 0.521 \\
\hline Regional lymph node & & & 0.008 \\
\hline$N(1)$ & 0.981 & $0.134-7.187$ & 0.985 \\
\hline$N(2)$ & 1.328 & $0.182-9.687$ & 0.780 \\
\hline$N(3)$ & 1.626 & $0.222-11.917$ & 0.632 \\
\hline Metastasis & & & 0.023 \\
\hline$M(1)$ & 0.575 & $0.282-1.453$ & 0.154 \\
\hline Histologic Grade & & & 0.157 \\
\hline $\mathrm{G}(1)$ & 0.284 & $0.030-2.734$ & 0.276 \\
\hline $\mathrm{G}(2)$ & 0.711 & $0.221-2.293$ & 0.568 \\
\hline Pathologic Stage & & & 0.002 \\
\hline$S(1)$ & 0.134 & $0.032-0.571$ & 0.007 \\
\hline$S(2)$ & 0.391 & $0.191-0.803$ & 0.011 \\
\hline$S(3)$ & 0.424 & $0.202-0.892$ & 0.024 \\
\hline E2F2 & 0.885 & $0.792-0.988$ & 0.030 \\
\hline
\end{tabular}

Table3 Multivariate analysis of overall survival in $366 \mathrm{GC}$ specimens. 


\begin{tabular}{|llll|}
\hline Variables & \multicolumn{3}{l|}{ Multivariate analysis } \\
\cline { 2 - 4 } & Hazard Ratio & $95 \% \mathrm{Cl}$ & P value \\
\hline Age(years) & 1.033 & $1.014-1.052$ & 0.001 \\
\hline Regional lymph node & & & 0.300 \\
\hline N(1) & 2.002 & $0.245-16.384$ & 0.518 \\
\hline N(2) & 1.459 & $0.190-11.209$ & 0.716 \\
\hline N(3) & 2.004 & $0.250-16.034$ & 0.512 \\
\hline Metastasis & & & 0.529 \\
\hline M(1) & 0.631 & $0.261-1.526$ & 0.307 \\
\hline Pathologic stage & & & 0.036 \\
\hline S(1) & 0.114 & $0.021-0.617$ & 0.012 \\
\hline S(2) & 0.29 & $0.093-0.902$ & 0.033 \\
\hline S(3) & 0.439 & $0.160-1.206$ & 0.010 \\
\hline E2F2 & 0.841 & $0.745-0.950$ & 0.005 \\
\hline
\end{tabular}

Figures 


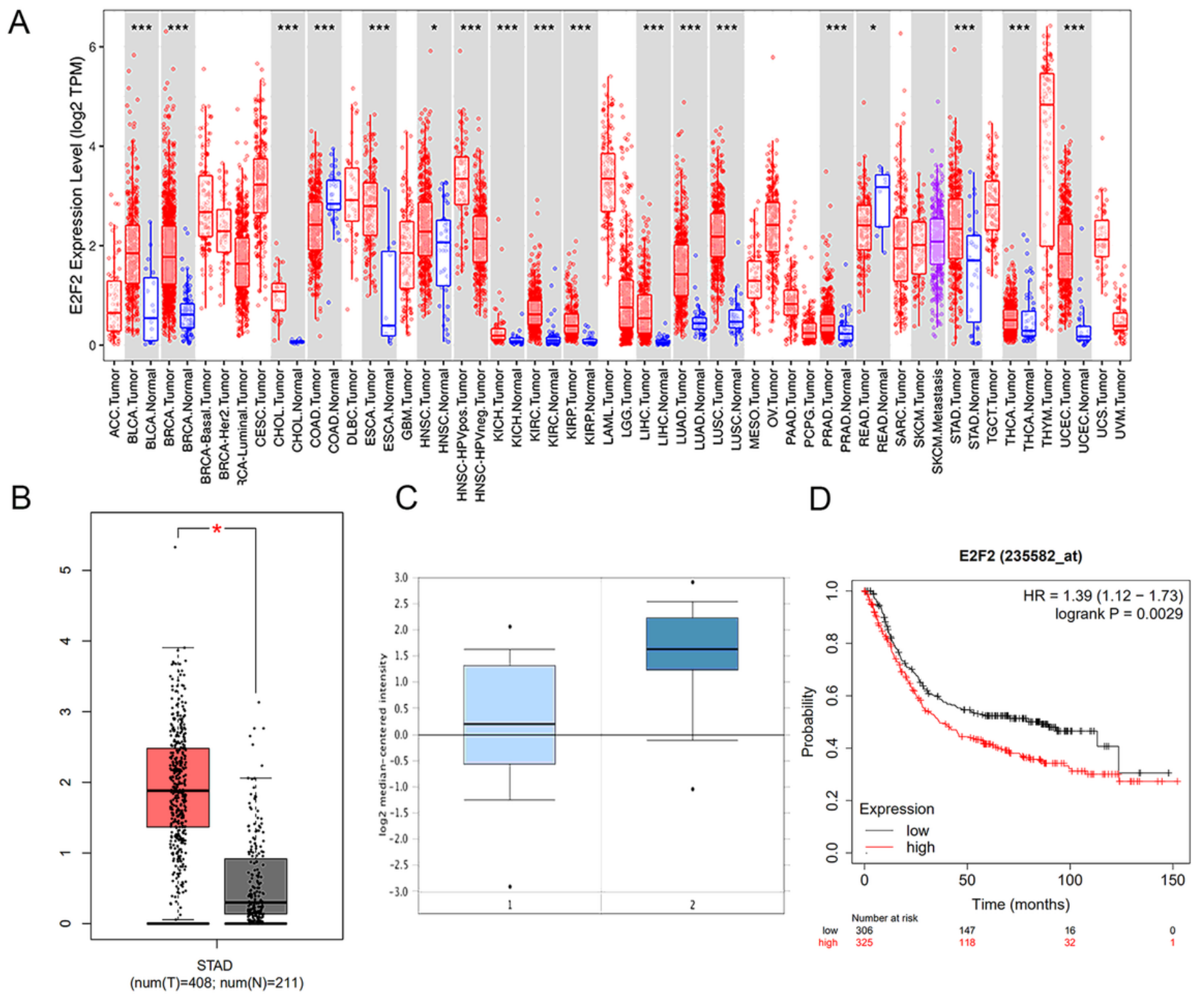

Figure 1

The expression and methylation of E2F2 in GC tissues and normal tissues revealed by bioinformatic analysis. (A) E2F2 expression levels of different tumor types in the TCGA database were detected by TIMER ( $\left.{ }^{\star} P<0.05,{ }^{\star} * P<0.01,{ }^{*} * P<0.001\right)$. (B and C) E2F2 mRNA is highly expressed in LGG tissues in GEPIA dataset (B) and Oncomine dataset. (D) Kaplan-Meier analysis of survival rates of GC patients with high E2F2 expression and GC patients with low E2F2 expression. 


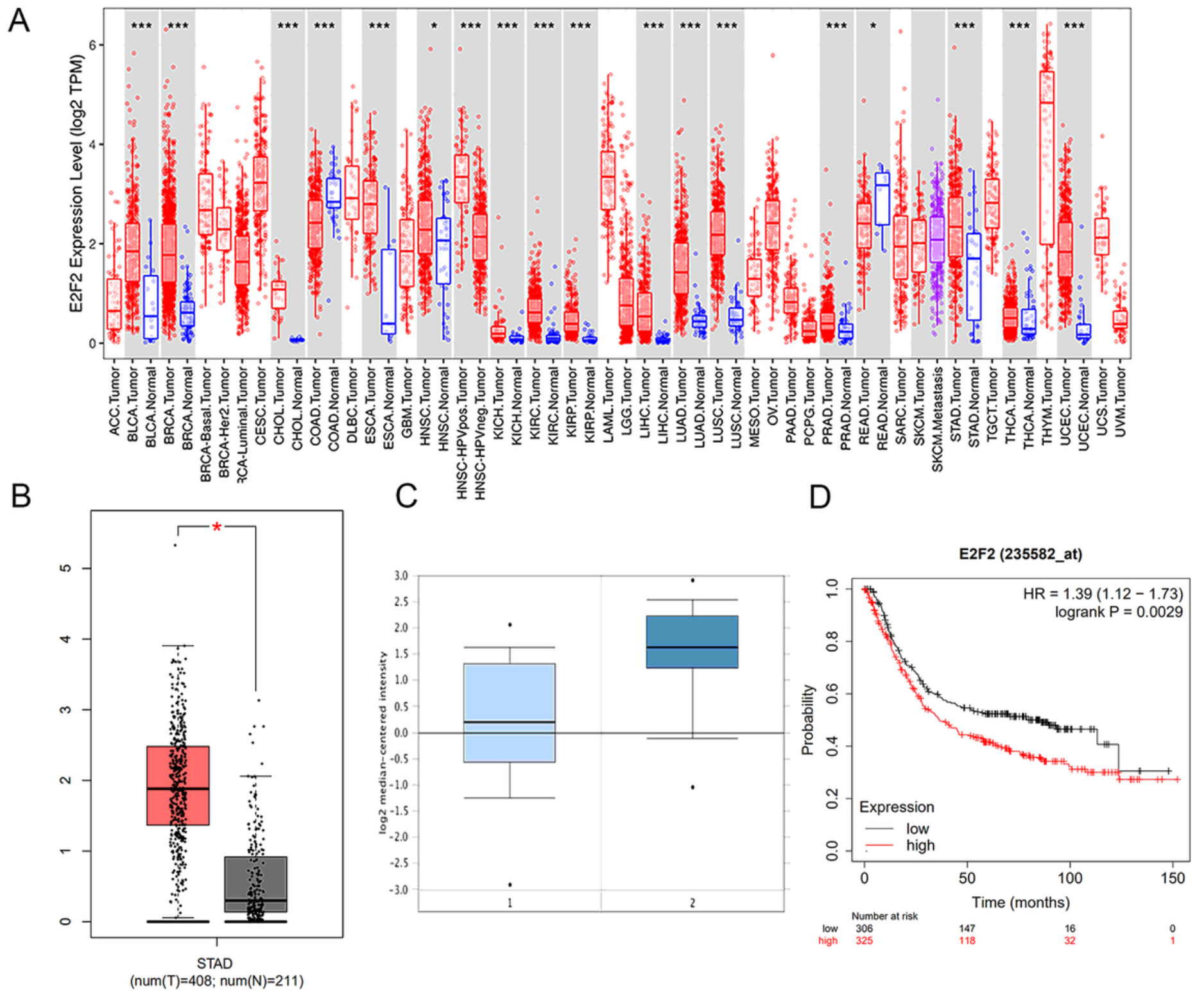

Figure 1

The expression and methylation of E2F2 in GC tissues and normal tissues revealed by bioinformatic analysis. (A) E2F2 expression levels of different tumor types in the TCGA database were detected by TIMER ( $\left.{ }^{\star} P<0.05,{ }^{\star} * P<0.01,{ }^{*} * P<0.001\right)$. (B and C) E2F2 mRNA is highly expressed in LGG tissues in GEPIA dataset (B) and Oncomine dataset. (D) Kaplan-Meier analysis of survival rates of GC patients with high E2F2 expression and GC patients with low E2F2 expression. 

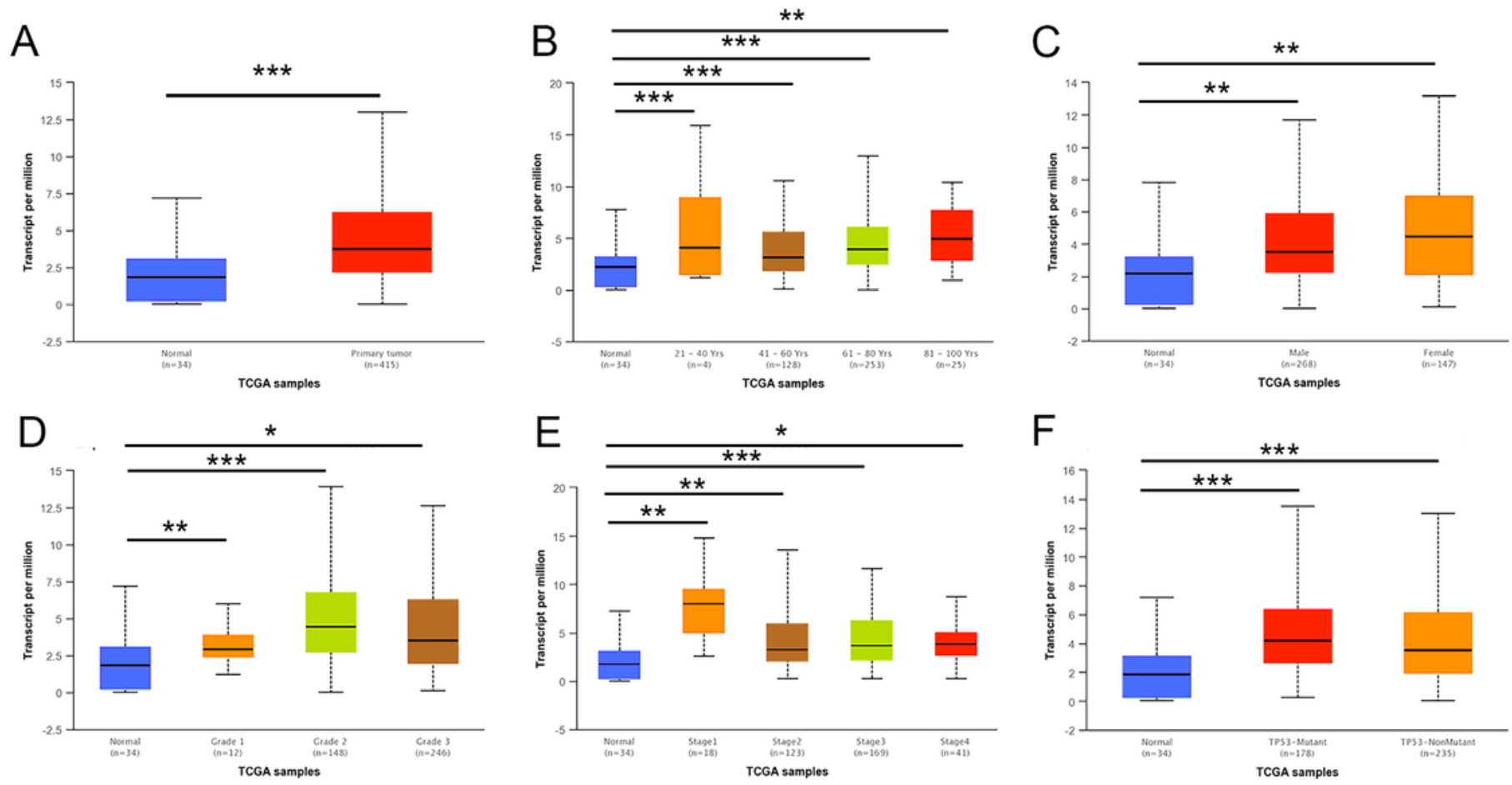

\section{Figure 2}

E2F2 transcription in subgroups of patients with GC, stratified based on gender, age and other criteria (UALCAN). Box-whisker plots showing the expression of E2F2 in sub groups of STAD samples. (A) Boxplot showing relative expression of E2F2 in normal and STAD samples. (B) Boxplot showing relative expression of E2F2 in normal individuals of any age or in STAD patients aged 21-40, 41-60, 61-80, or 81$100 \mathrm{yr}$. (C) Boxplot showing relative expression of E2F2 in normal individuals of either gender and male or female STAD patients, respectively. (D) Boxplot showing relative expression of E2F2 in normal individuals or in STAD patients in stages 1, 2, 3 or 4. (E) Boxplot showing relative expression of E2F2 in normal individuals or STAD patients with grade 1, 2, 3 or 4 tumors. (F) Boxplot showing relative expression of E2F2 based on TP53 mutation status. The central mark is the median; the edges of the box are the 25th and 75th percentiles. The t-test was used to estimate the significance of difference in gene expression levels between groups. *, $p<0.05 ; * \star, p<0.01 ; * * *, p<0.001$. 

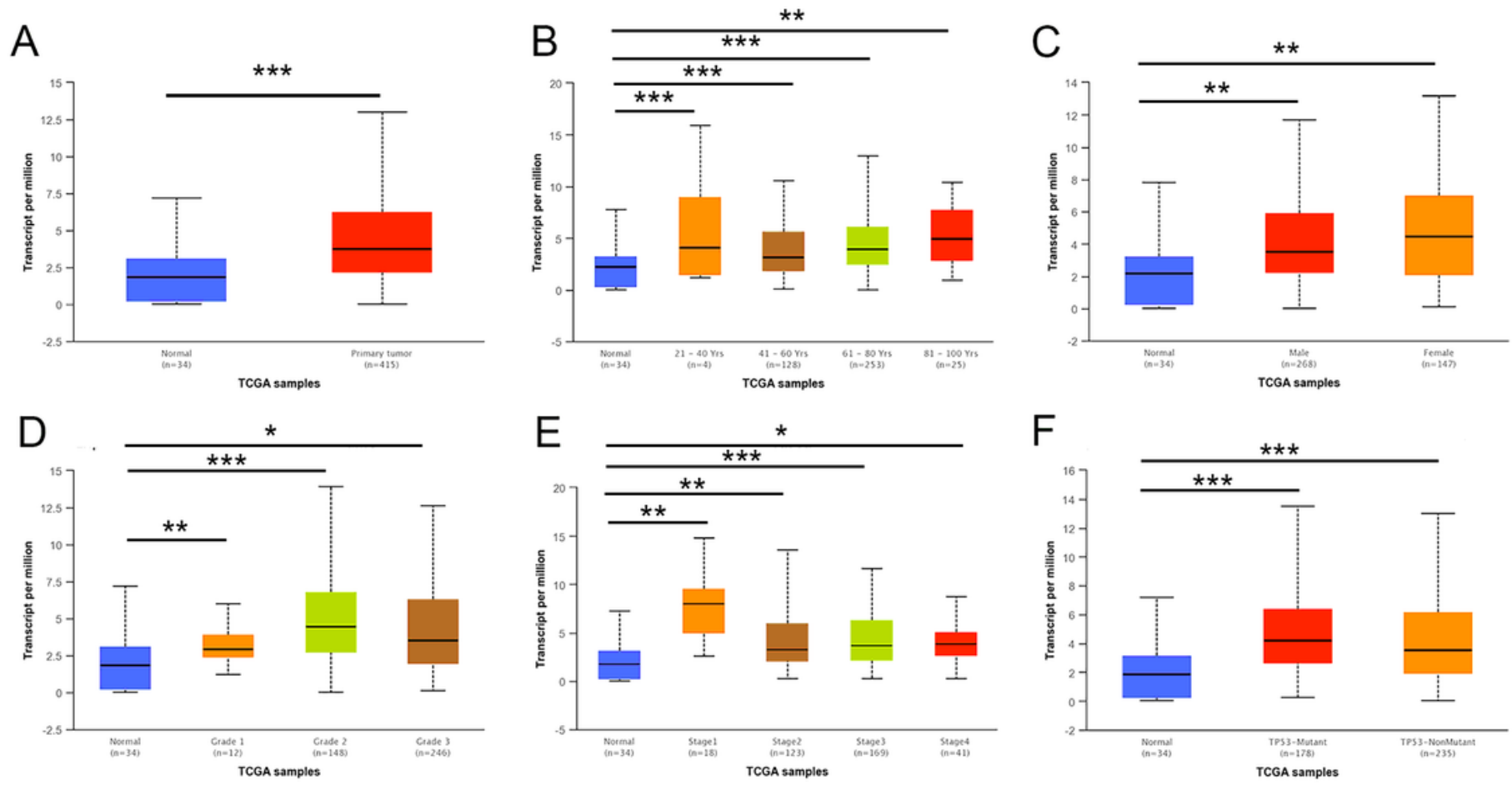

\section{Figure 2}

E2F2 transcription in subgroups of patients with GC, stratified based on gender, age and other criteria (UALCAN). Box-whisker plots showing the expression of E2F2 in sub groups of STAD samples. (A) Boxplot showing relative expression of E2F2 in normal and STAD samples. (B) Boxplot showing relative expression of E2F2 in normal individuals of any age or in STAD patients aged 21-40, 41-60, 61-80, or 81$100 \mathrm{yr}$. (C) Boxplot showing relative expression of E2F2 in normal individuals of either gender and male or female STAD patients, respectively. (D) Boxplot showing relative expression of E2F2 in normal individuals or in STAD patients in stages 1, 2, 3 or 4. (E) Boxplot showing relative expression of E2F2 in normal individuals or STAD patients with grade 1, 2, 3 or 4 tumors. (F) Boxplot showing relative expression of E2F2 based on TP53 mutation status. The central mark is the median; the edges of the box are the 25th and 75th percentiles. The t-test was used to estimate the significance of difference in gene expression levels between groups. *, $p<0.05 ; * \star, p<0.01 ; * * *, p<0.001$. 
A

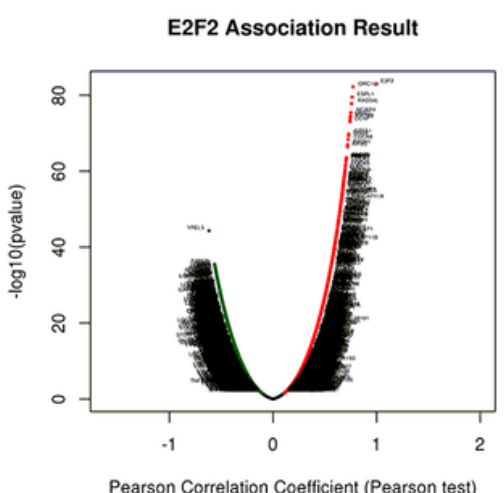

B

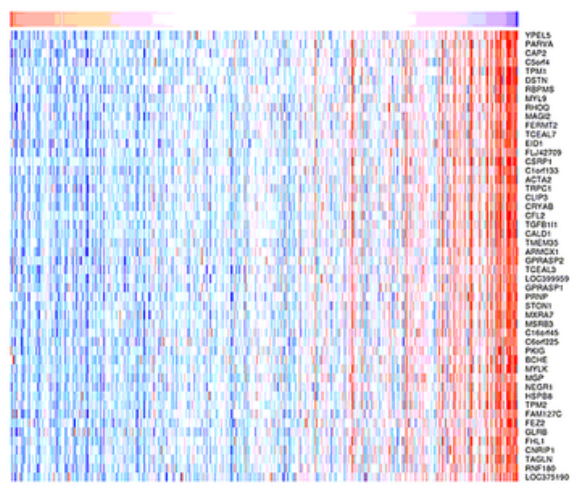

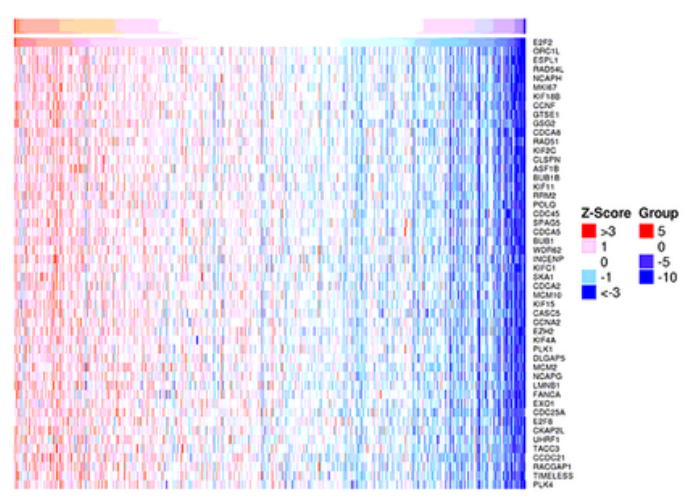

C

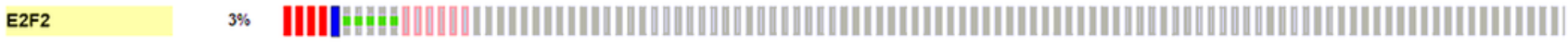

Genetic Alteration

If Missense Mutation (unknown significance)

| Ampirifation

| Deep Deletion || mRNA High || No atterations

D

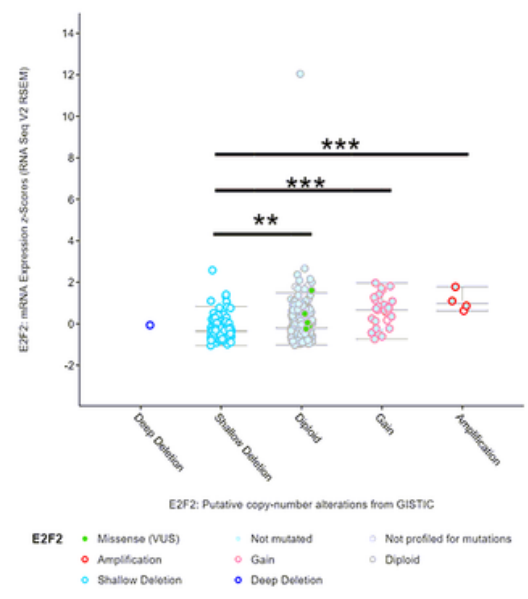

$\mathrm{E}$

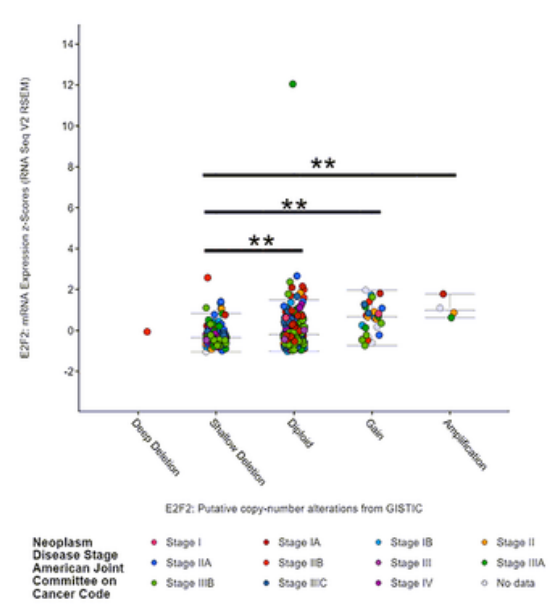

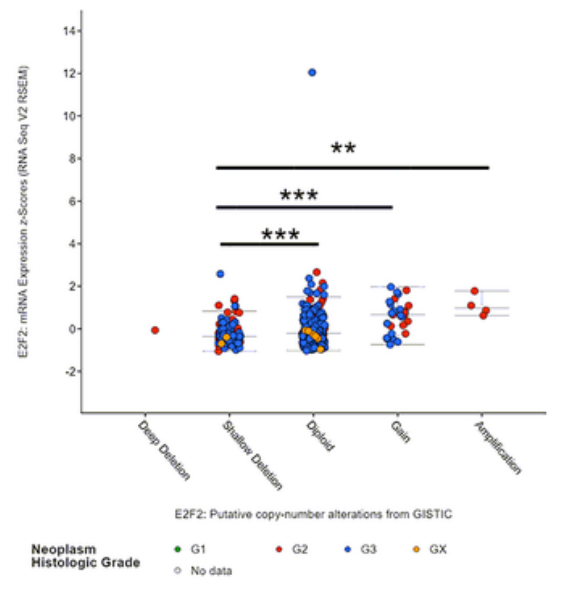

Figure 3

E2F2 co-expression genes (LinkedOmics) and genomic alterations (cBioPortal) in GC. (A) The global E2F2 highly correlated genes identified by Pearson test in STAD cohort. (B) Heat maps showing top 50 genes positively and negatively correlated with E2F2 in STAD. Red indicates positively correlated genes and blue indicates negatively correlated genes. (C) OncoPrint of E2F2 alterations in STAD cohort. The different types of genetic alterations are highlighted in different colors. (D) E2F2 expression in different E2F2 CNV groups. (E and F) Distribution of E2F2 CNV frequency in different stage and grade subgroups. ${ }^{\star} P<0.05,{ }^{\star *} \mathrm{P}<0.01,{ }^{\star * *} \mathrm{P}<0.001$. 
A

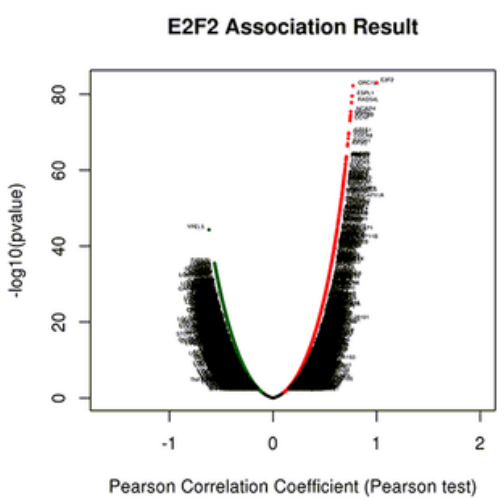

B

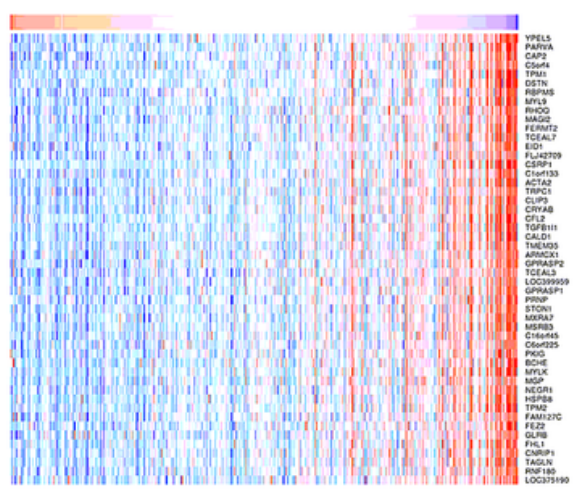

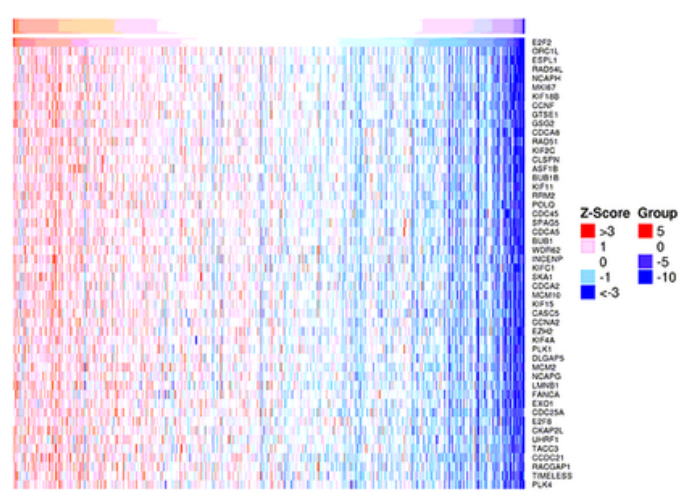

C

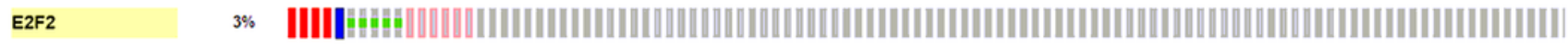

Genetic Alteration

If Missense Mutation (unknown significance)

| Amplification

| Deep Deletion || mRNA High || No atterations

D

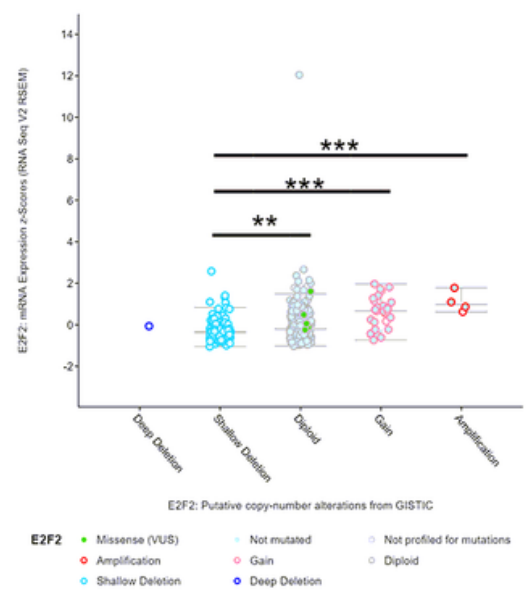

E

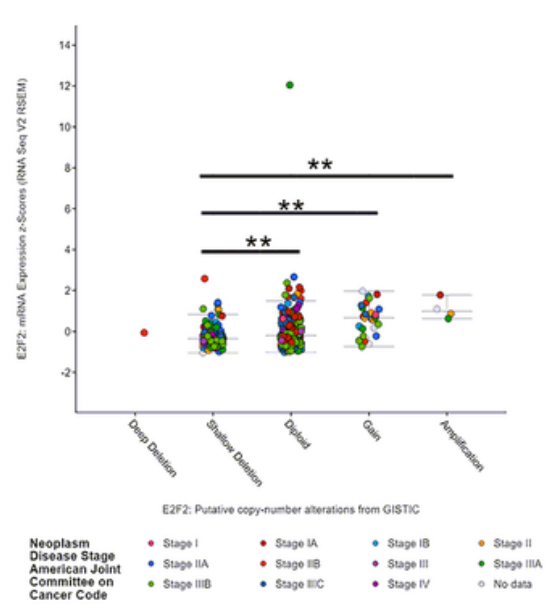

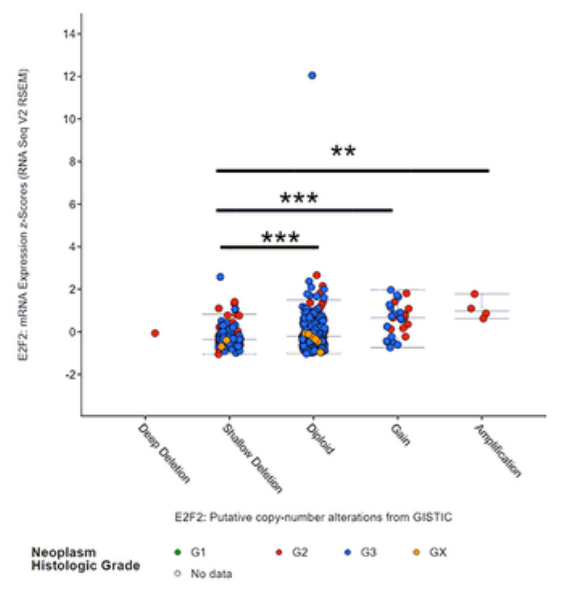

\section{Figure 3}

E2F2 co-expression genes (LinkedOmics) and genomic alterations (cBioPortal) in GC. (A) The global E2F2 highly correlated genes identified by Pearson test in STAD cohort. (B) Heat maps showing top 50 genes positively and negatively correlated with E2F2 in STAD. Red indicates positively correlated genes and blue indicates negatively correlated genes. (C) OncoPrint of E2F2 alterations in STAD cohort. The different types of genetic alterations are highlighted in different colors. (D) E2F2 expression in different E2F2 CNV groups. (E and F) Distribution of E2F2 CNV frequency in different stage and grade subgroups. ${ }^{\star} P<0.05,{ }^{\star *} \mathrm{P}<0.01,{ }^{\star * *} \mathrm{P}<0.001$. 


\section{A}

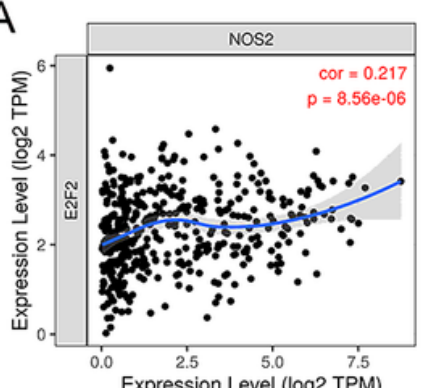

C

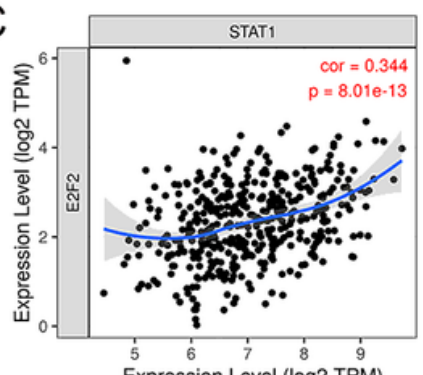

$\mathrm{E}$

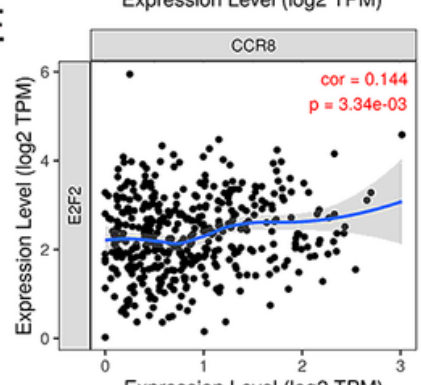

Expression Level (log2 TPM)
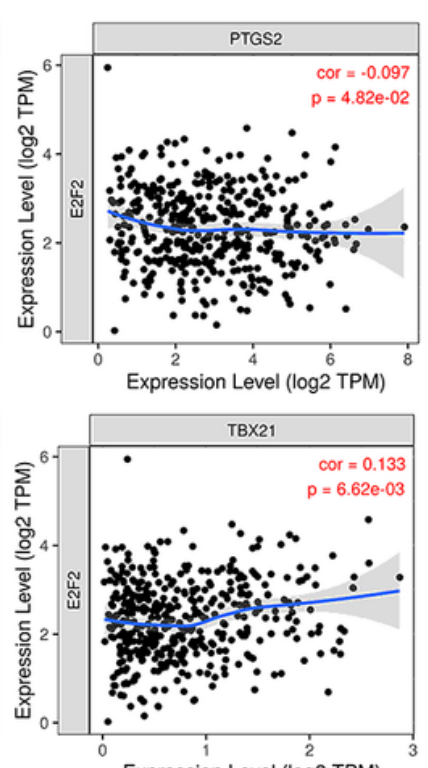

Expression Level (log2 TPM)

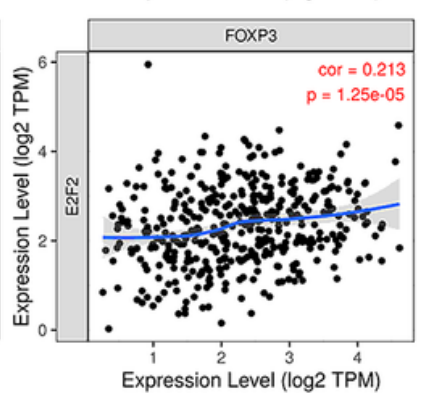

B
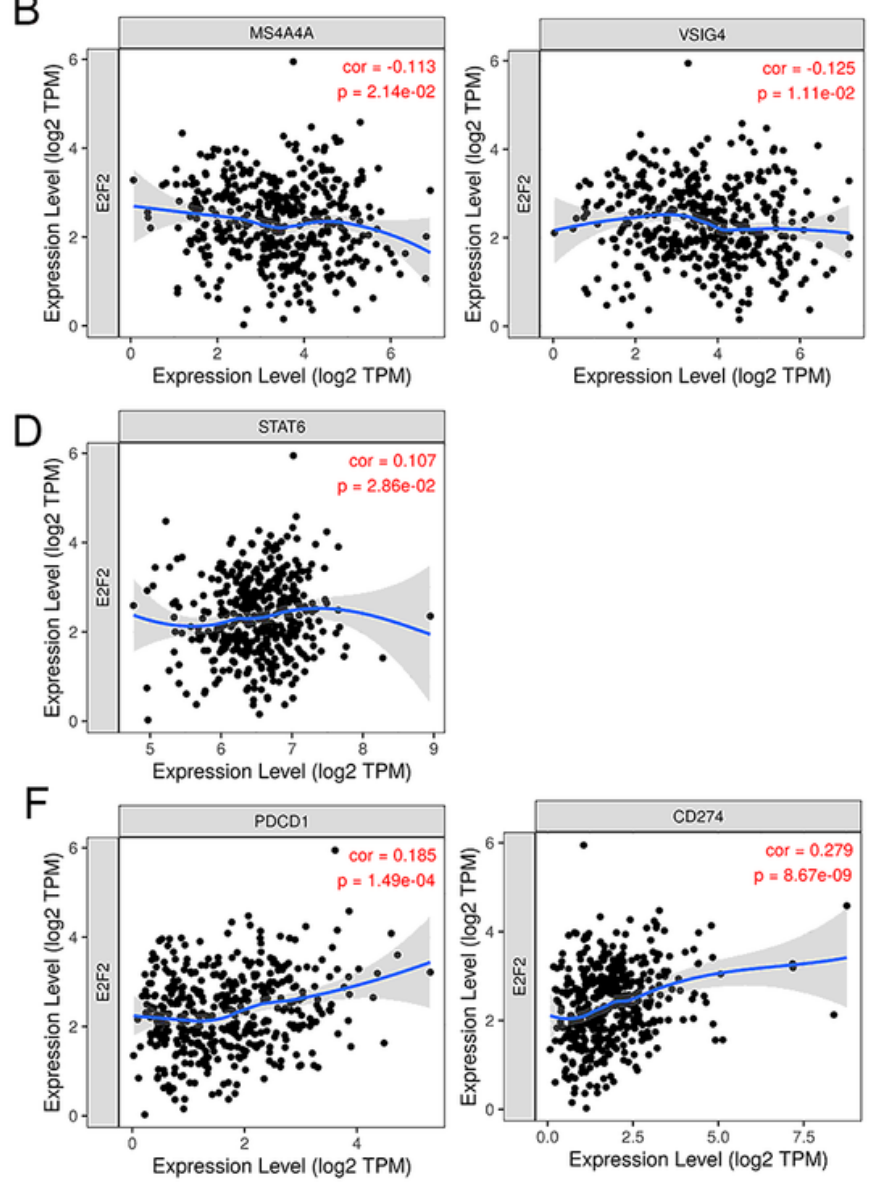

G

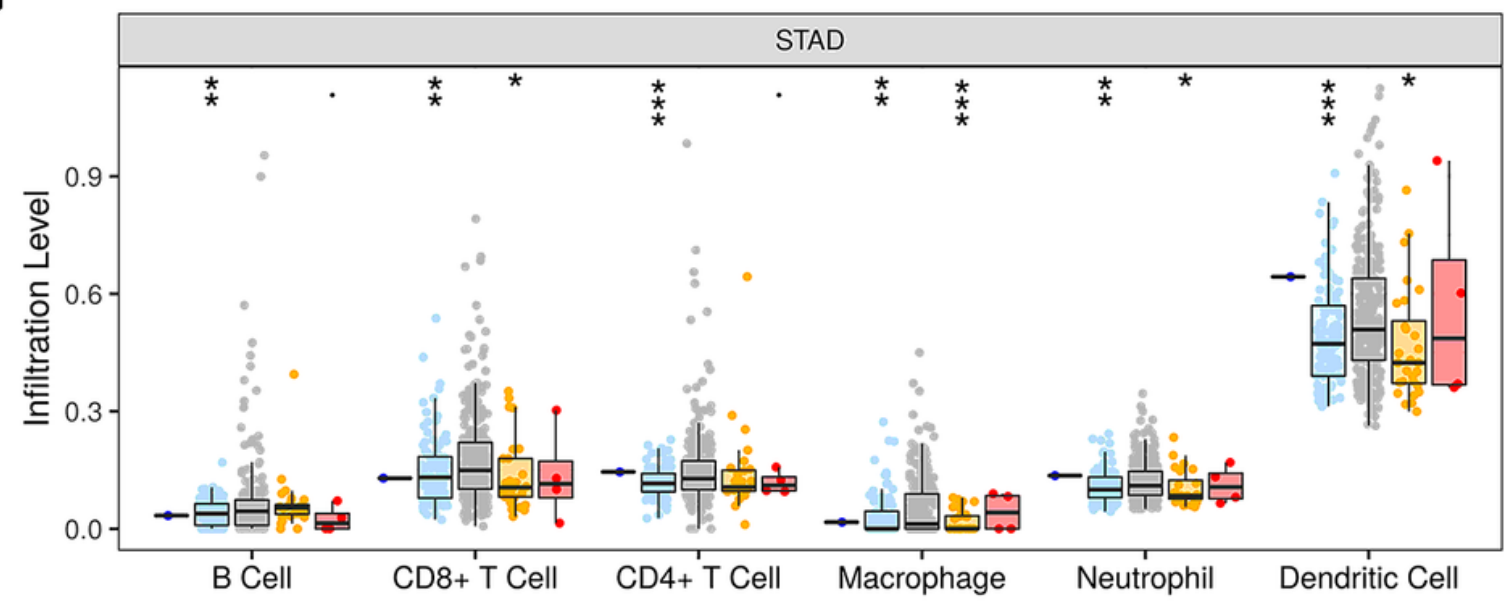

Copy Number

柬 Deep Deletion

审 Arm-level Deletion

审 Diploid/Normal

蒋 Arm-level Gain

审 High Amplication

\section{Figure 4}

The expression of E2F2 was related to a panel of gene markers of immune cells, including M1 cell (A), M2 cell (B), Th1 cell (C), Th2 cell (D) , Treg cell (E) and PD-1/PD-L1 (F). (G) E2F2 CNV affects the infiltrating levels of $B$ cells, $C D 8+T$ cells $\square C D 4+T$ cells, macrophages, neutrophils and dendritic cells in GC 
A
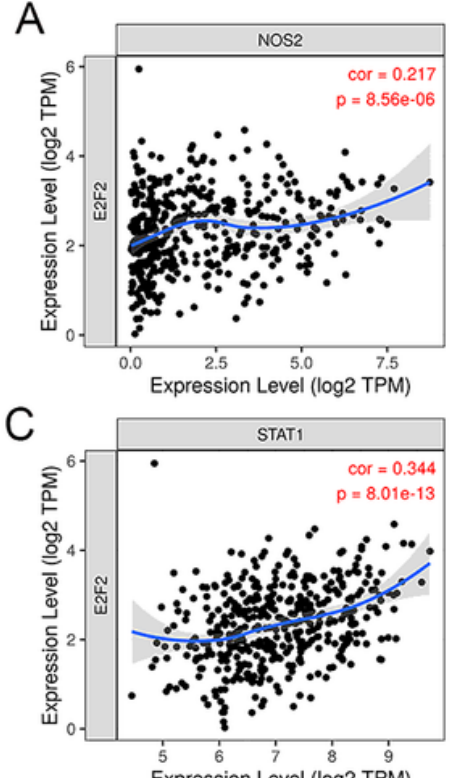

E

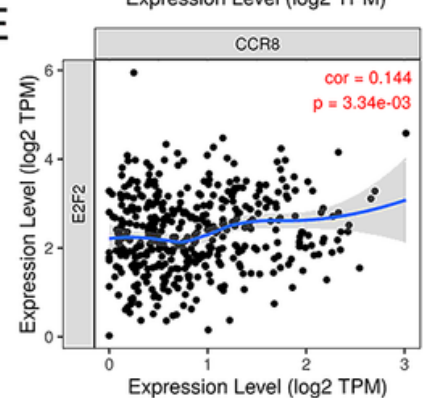

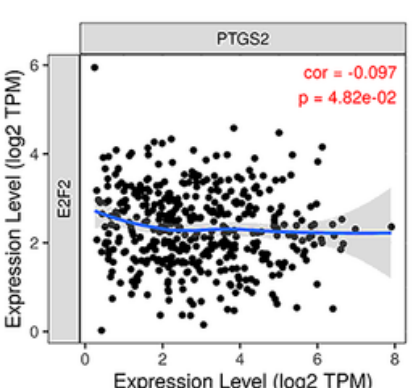

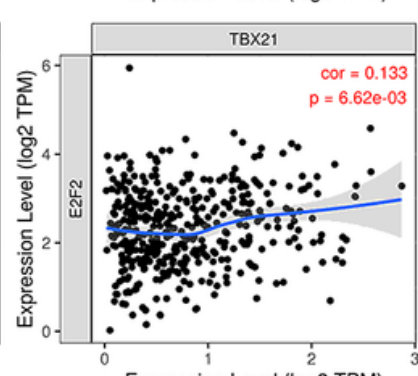

Expression Level (log2 TPM)

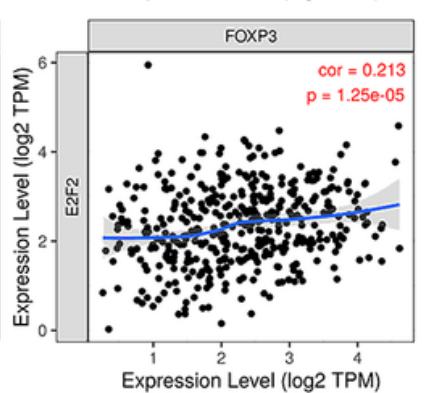

B
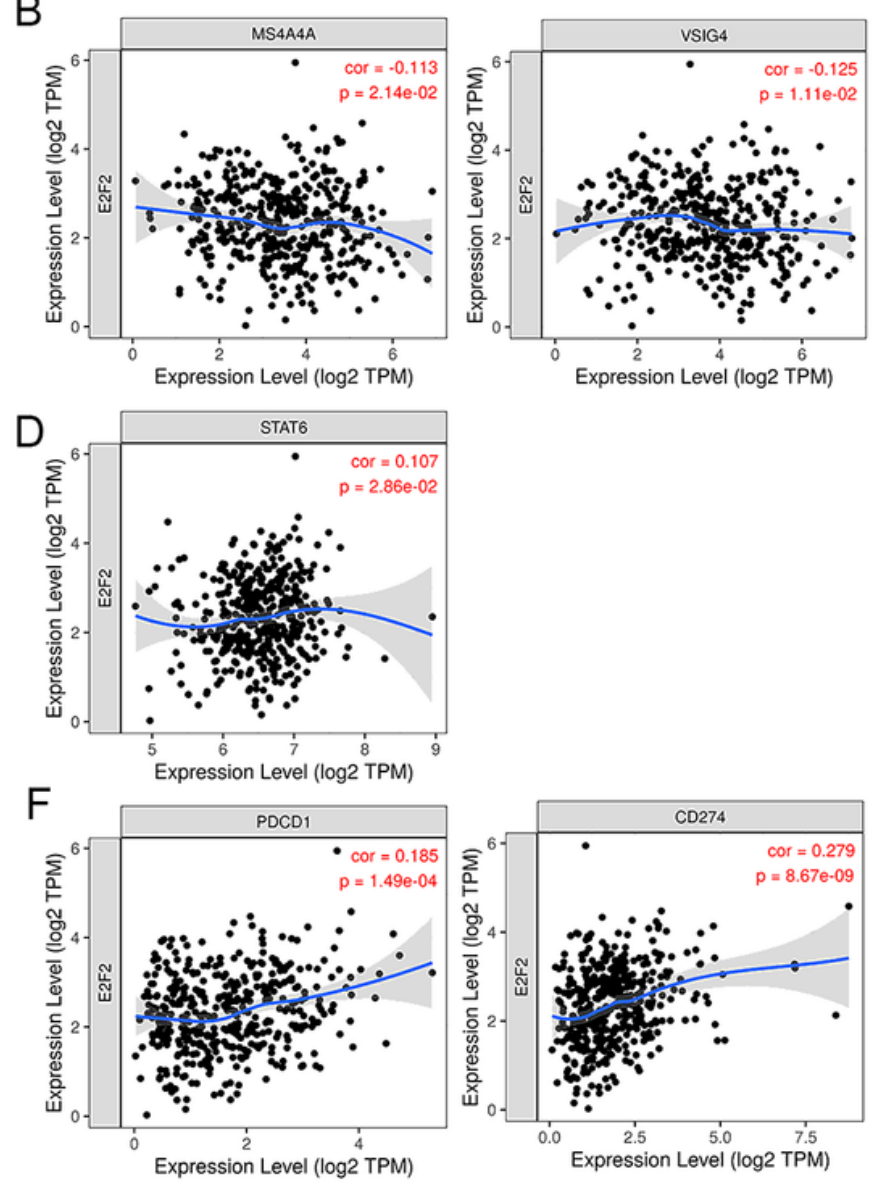

G

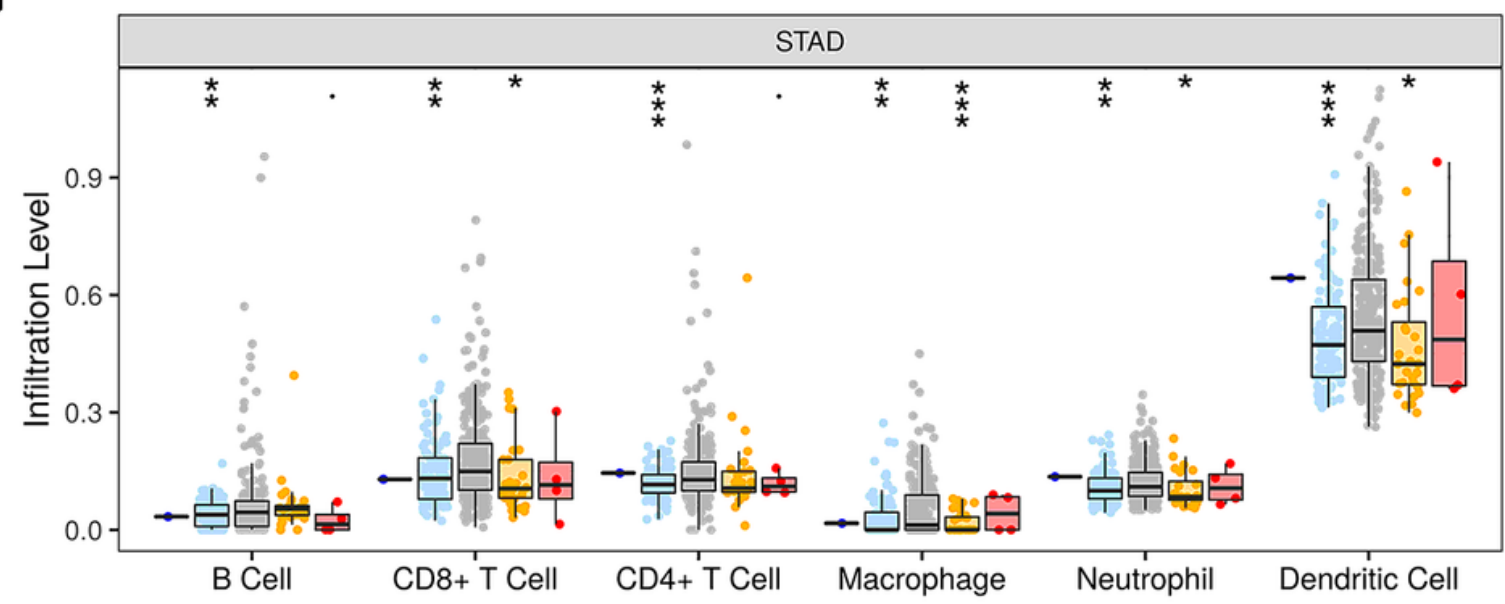

Copy Number

柬 Deep Deletion

审 Arm-level Deletion

审 Diploid/Normal

审 Arm-level Gain

审 High Amplication

\section{Figure 4}

The expression of E2F2 was related to a panel of gene markers of immune cells, including M1 cell (A), M2 cell (B), Th1 cell (C), Th2 cell (D) , Treg cell (E) and PD-1/PD-L1 (F). (G) E2F2 CNV affects the infiltrating levels of $B$ cells, $C D 8+T$ cells $\square C D 4+T$ cells, macrophages, neutrophils and dendritic cells in GC 
A

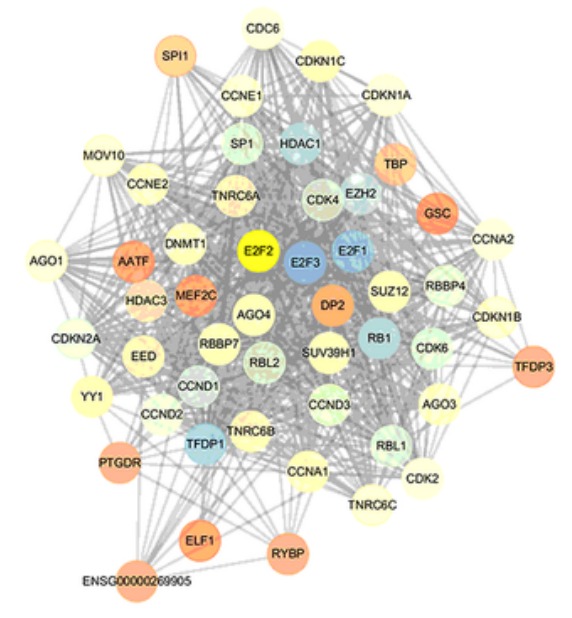

C
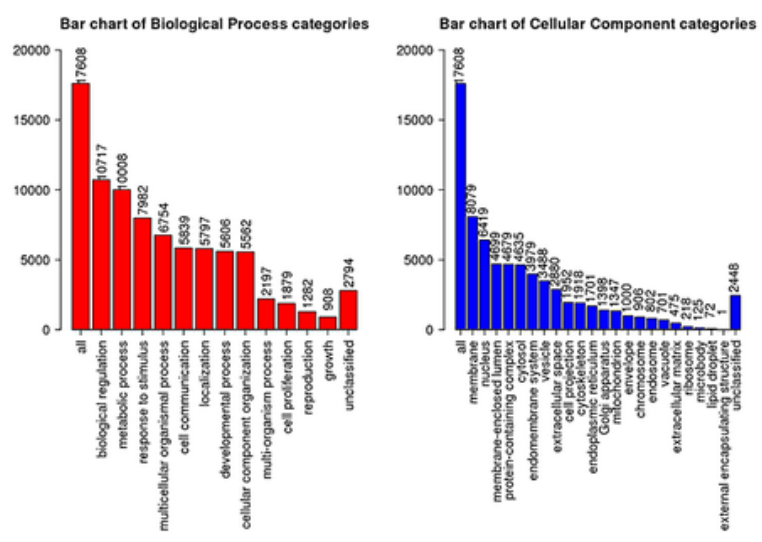

D

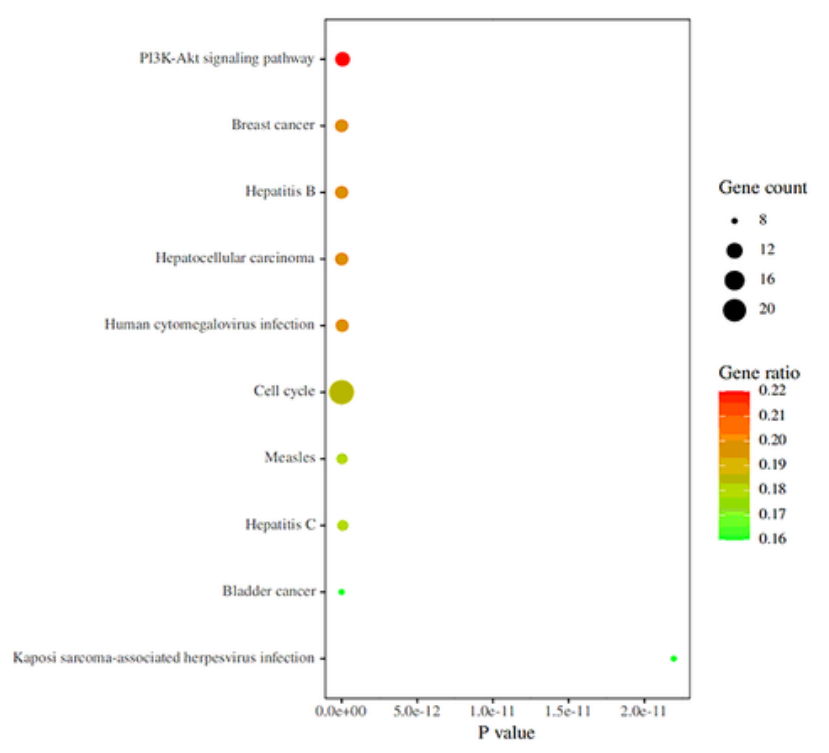

B
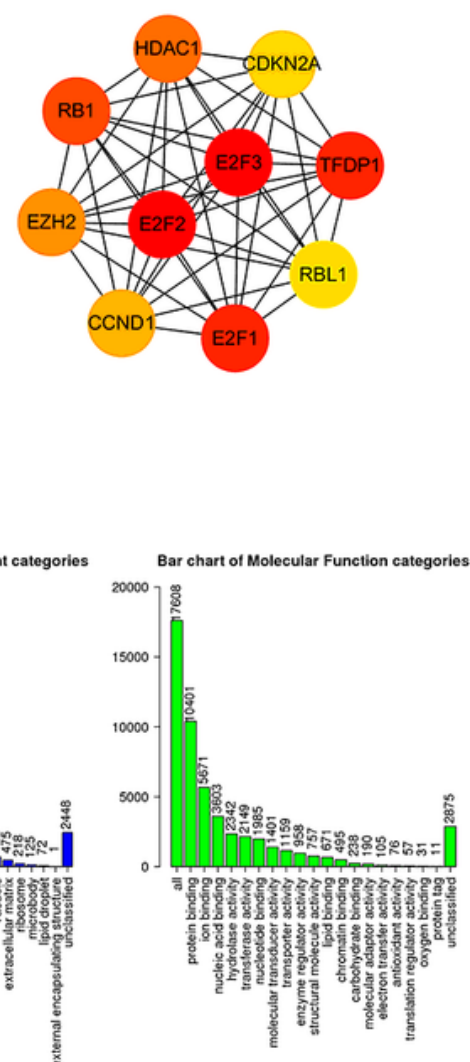

\section{Figure 5}

Protein-protein interaction (PPI) network construction and gene enrichment analyses. (A) Network of E2F2 and its 50 frequently altered neighbor genes was constructed. (B) Hub genes were screened from the PPI network using the Closeness, Degree and MCC methods. (C) The functional enrichment histogram of important modules. Each biological process, cellular component and molecular function category is represented by a red, blue and green bar, respectively. The height represents the number of IDs in the user 
list and in the category. (D) Pathways enrichment map of E2F2 and its 50 frequently altered neighbor genes. The top 20 terms with the largest number of enriched genes were selected.

A

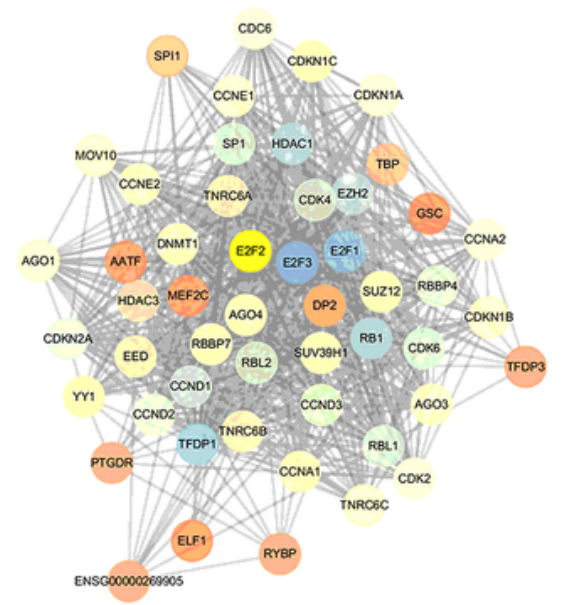

c
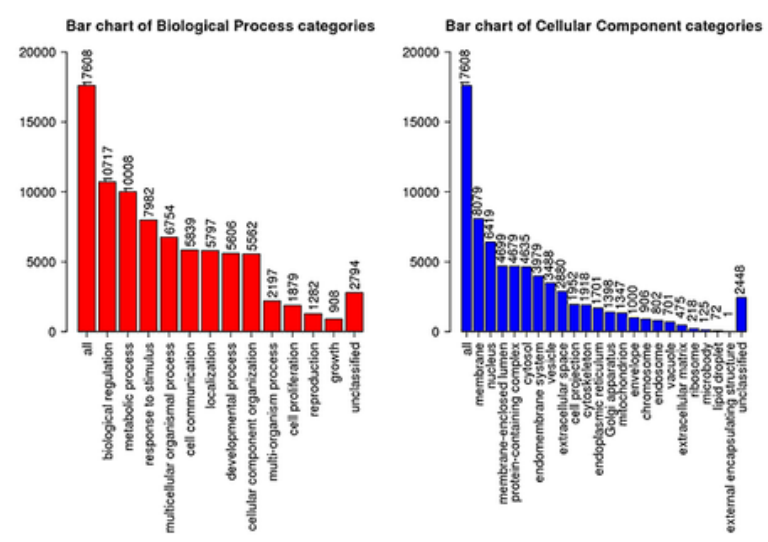

D

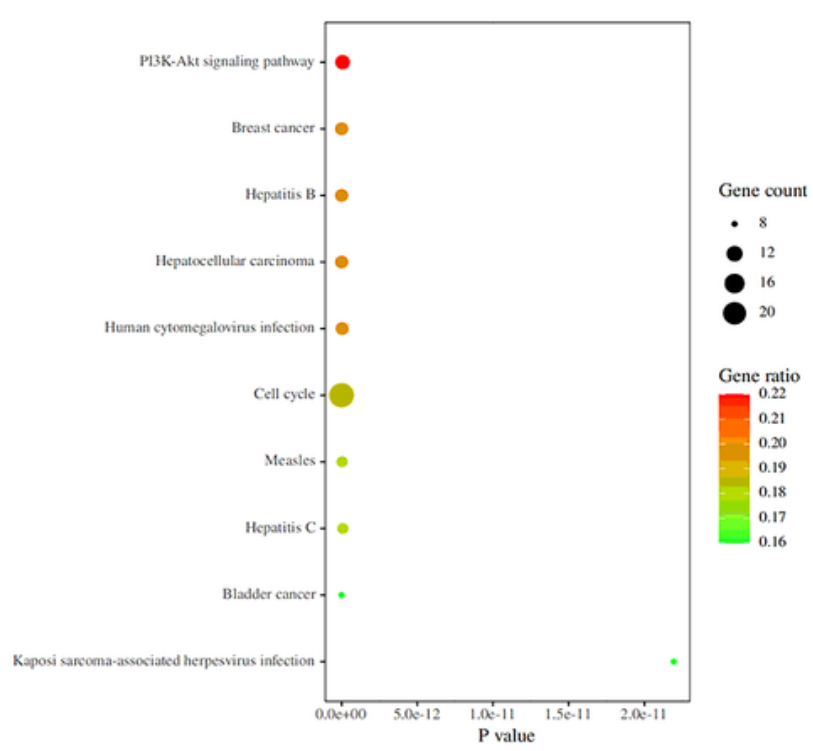

B
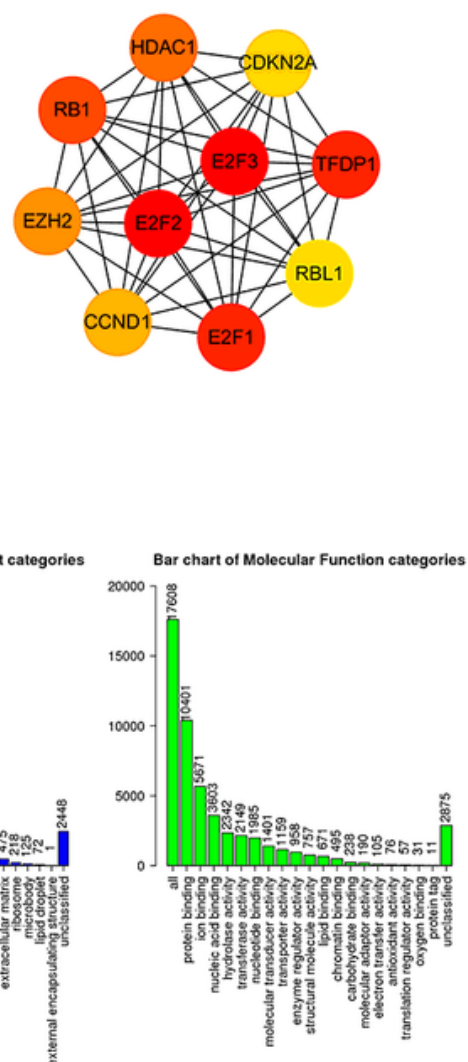

ene count

12

16

e ratio 0.22

\section{Figure 5}

Protein-protein interaction (PPI) network construction and gene enrichment analyses. (A) Network of E2F2 and its 50 frequently altered neighbor genes was constructed. (B) Hub genes were screened from the PPI network using the Closeness, Degree and MCC methods. (C) The functional enrichment histogram of 
important modules. Each biological process, cellular component and molecular function category is represented by a red, blue and green bar, respectively. The height represents the number of IDs in the user list and in the category. (D) Pathways enrichment map of E2F2 and its 50 frequently altered neighbor genes. The top 20 terms with the largest number of enriched genes were selected.
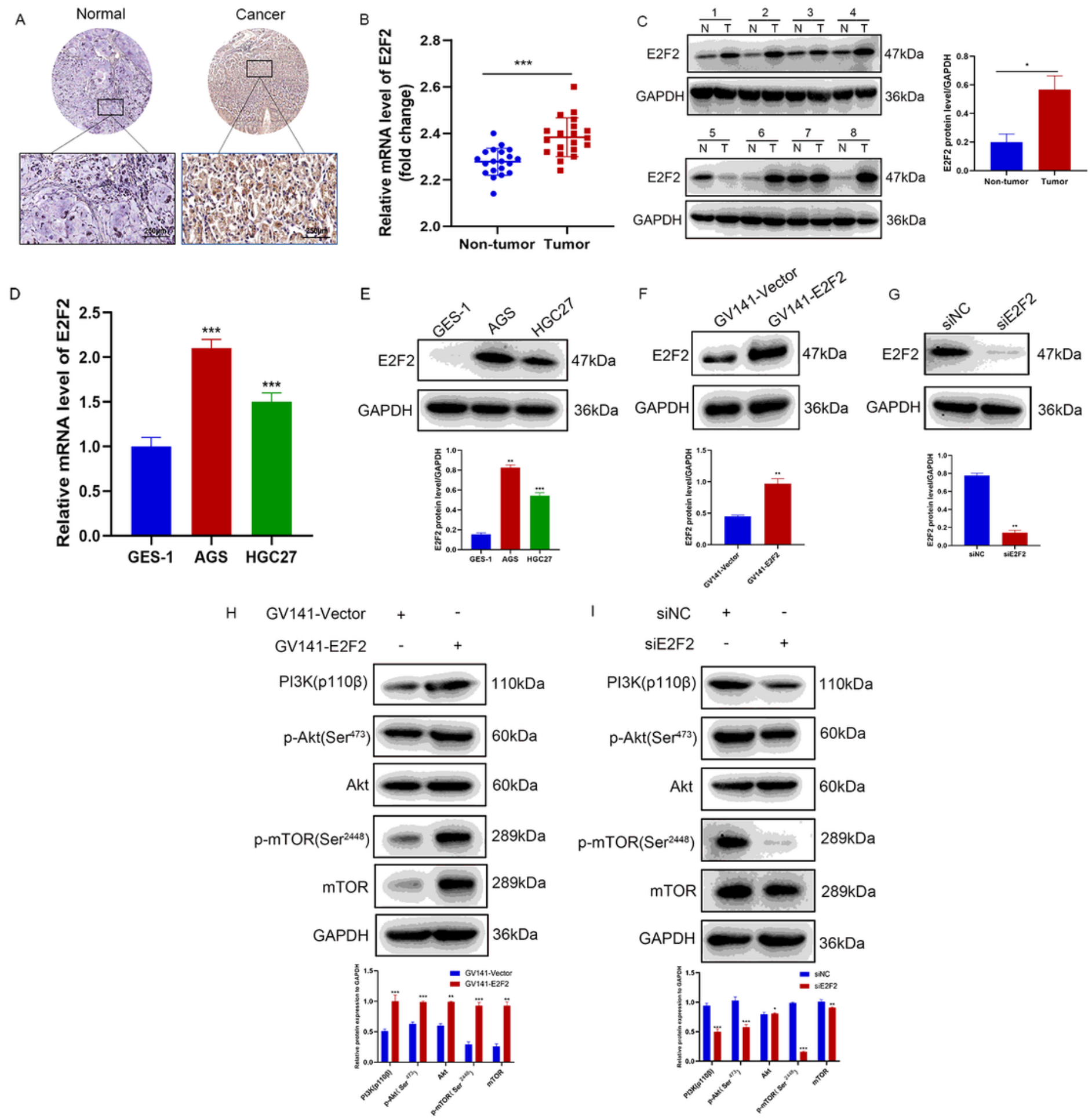

Figure 6 
E2F2 is a regulator of the PI3K/Akt/mTOR pathway. (A) Representative images of E2F2 TMA analysis in GC tissues and adjacent tissues. Scale bar, 250 $\mu \mathrm{m}$. (B) Quantitative PCR analysis of E2F2 mRNA expression in GC and non-tumor gastric tissues in our patient cohort. E2F2 mRNA expression levels were normalized according to glyceraldehyde 3-phosphate dehydrogenase expression levels ( $n=20$ per group). (C) Western blotting analysis of E2F2 protein expression in GC (T) and non-tumor gastric tissues $(\mathrm{N})$. E2F2 protein expression levels were normalized according to $\beta$-actin expression levels ( $n=8$ per group). (D) Quantitative PCR (qPCR) analysis of E2F2 basal mRNA expression in three cell lines. E2F2 mRNA expression levels were normalized according to the glyceraldehyde 3-phosphate dehydrogenase (GAPDH) expression levels. (E) Western blotting analysis of E2F2 basal protein expression in the three cell lines; $\beta$ actin was used as a loading control. (F) Western blotting analysis of E2F2 protein expression in HGC27 cells transfected with GV141-Vector and GV141-E2F2 for $24 \mathrm{~h}$. $\beta$-Actin was used as a loading control. (G) Western blotting analysis of E2F2 protein expression in AGS cells transfected with siE2F2 for $24 \mathrm{~h}$. $\beta$ Actin was used as a loading control. $(\mathrm{H})$ Western blotting analysis of the PI3K 110, p-AKT, AKT, p-mTOR, and mTOR protein expression in HGC27 cells transfected with GV141-Vector and GV141-E2F2 for $24 \mathrm{~h}$. $\beta$ Actin was used as a loading control. (I) Western blotting analysis of PI3K 110ß, p-AKT, AKT, p-mTOR and mTOR protein expression in AGS cells transfected with siNC and siE2F2 for $24 \mathrm{~h}$. $\beta$-Actin was used as a loading control. Data are presented as mean \pm S.D. from three independent experiments. ${ }^{*} P<0.05$, ${ }^{\star *} \mathrm{P}<$ $0.01,{ }^{* \star *} \mathrm{P}<0.001$. 

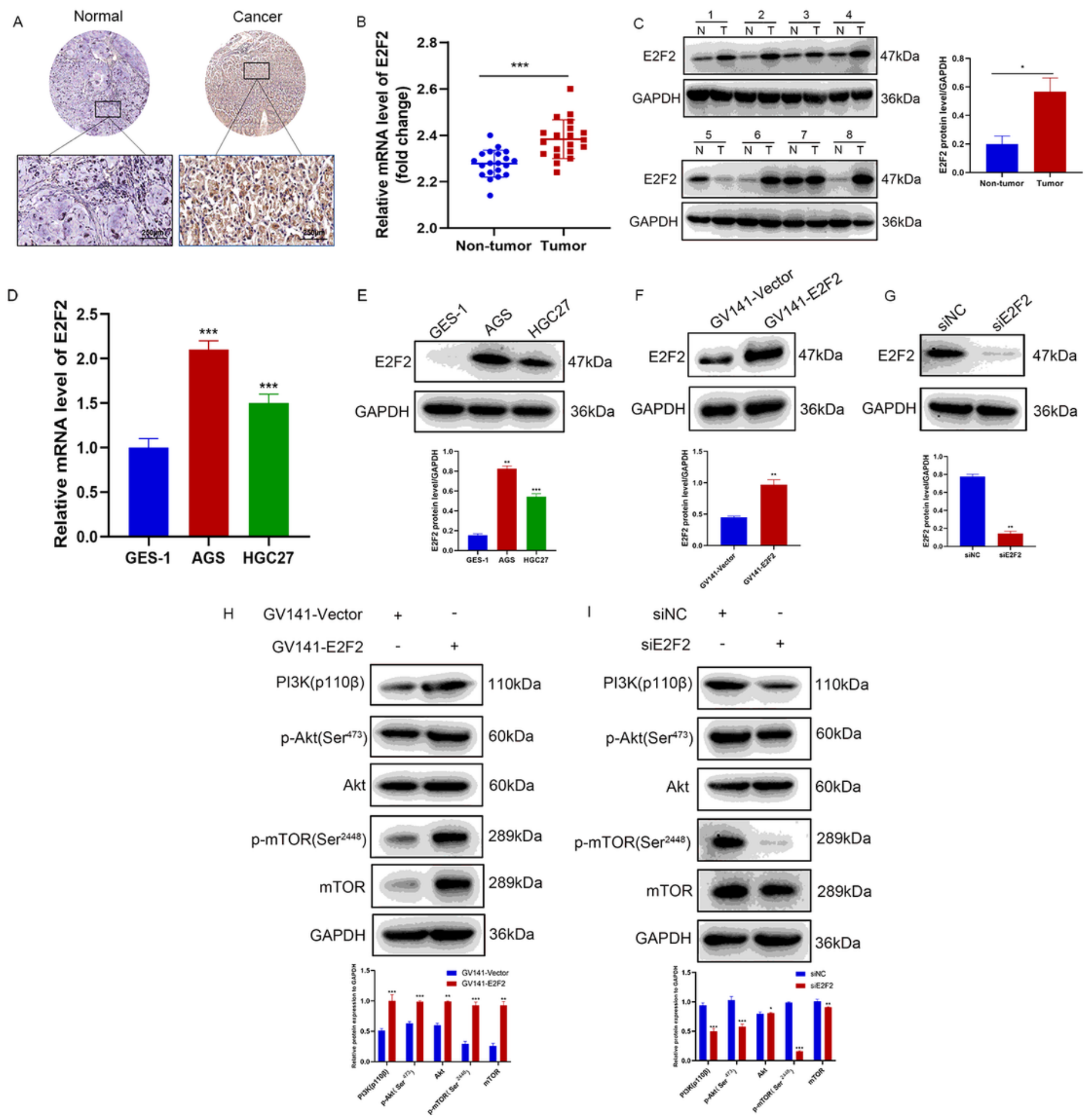

\section{Figure 6}

E2F2 is a regulator of the PI3K/Akt/mTOR pathway. (A) Representative images of E2F2 TMA analysis in GC tissues and adjacent tissues. Scale bar, 250 $\mu \mathrm{m}$. (B) Quantitative PCR analysis of E2F2 mRNA expression in GC and non-tumor gastric tissues in our patient cohort. E2F2 mRNA expression levels were normalized according to glyceraldehyde 3-phosphate dehydrogenase expression levels ( $\mathrm{n}=20$ per group). (C) Western blotting analysis of E2F2 protein expression in GC (T) and non-tumor gastric tissues $(\mathrm{N})$. 
E2F2 protein expression levels were normalized according to $\beta$-actin expression levels ( $n=8$ per group). (D) Quantitative PCR (qPCR) analysis of E2F2 basal mRNA expression in three cell lines. E2F2 mRNA expression levels were normalized according to the glyceraldehyde 3-phosphate dehydrogenase (GAPDH) expression levels. (E) Western blotting analysis of E2F2 basal protein expression in the three cell lines; $\beta$ actin was used as a loading control. (F) Western blotting analysis of E2F2 protein expression in HGC27 cells transfected with GV141-Vector and GV141-E2F2 for $24 \mathrm{~h}$. $\beta$-Actin was used as a loading control. (G) Western blotting analysis of E2F2 protein expression in AGS cells transfected with siE2F2 for $24 \mathrm{~h}$. $\beta$ Actin was used as a loading control. $(H)$ Western blotting analysis of the PI3K 110, p-AKT, AKT, p-mTOR, and mTOR protein expression in HGC27 cells transfected with GV141-Vector and GV141-E2F2 for $24 \mathrm{~h}$. $\beta$ Actin was used as a loading control. (I) Western blotting analysis of PI3K 110ß, p-AKT, AKT, p-mTOR and mTOR protein expression in AGS cells transfected with siNC and siE2F2 for $24 \mathrm{~h}$. $\beta$-Actin was used as a loading control. Data are presented as mean \pm S.D. from three independent experiments. ${ }^{*} P<0.05, * * P<$ $0.01,{ }^{\star} * \star \mathrm{P}<0.001$. 
A
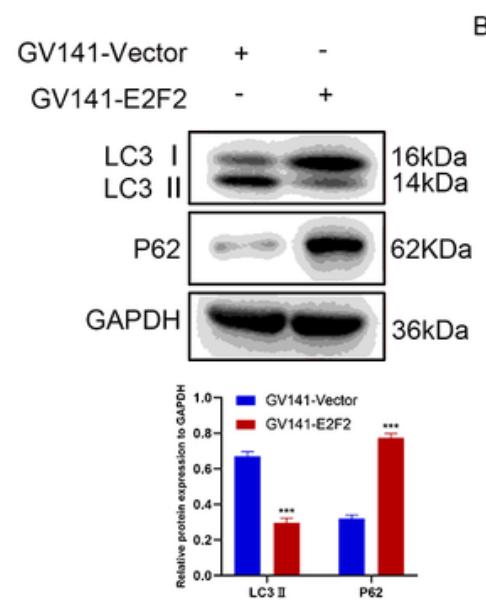

D

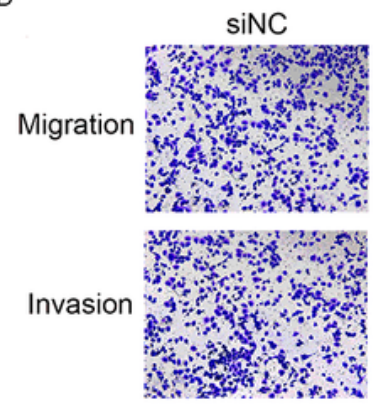

B
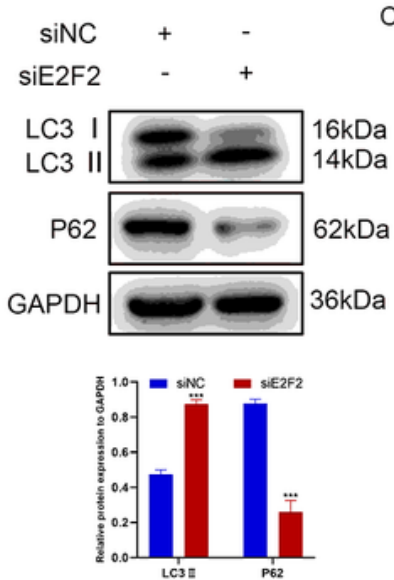

C
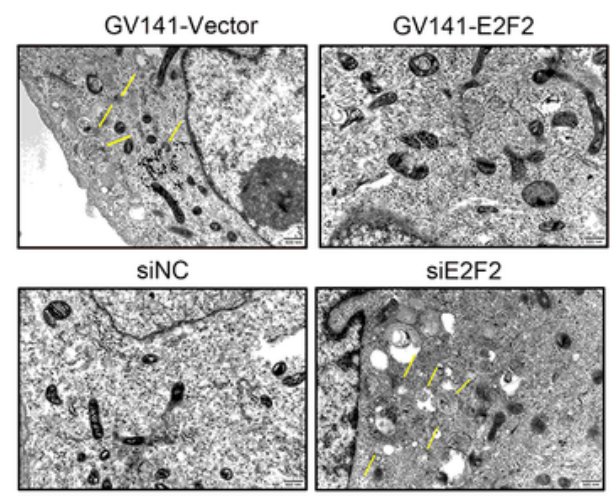

E

SiE2F2
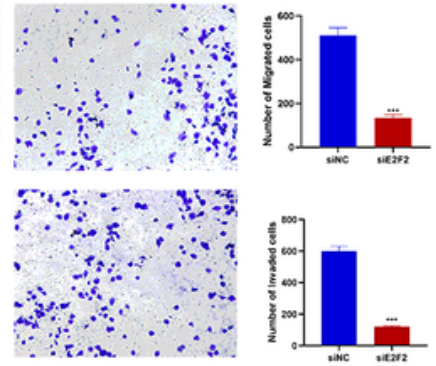

$24 h$

siNC

siE2F2

Oh
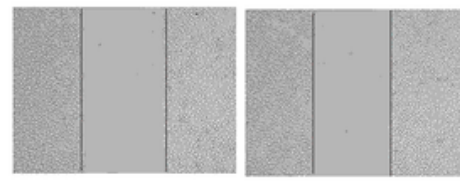

$\because 48$
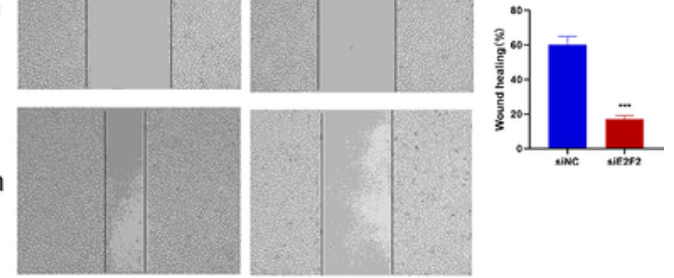

F

siNC

SiE2F2

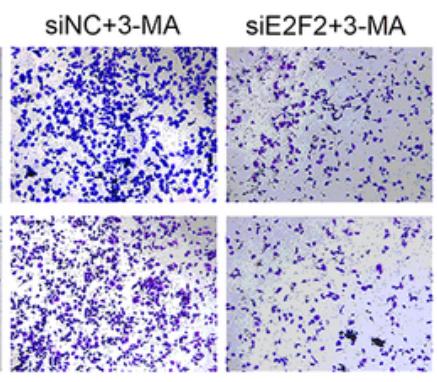

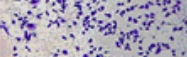

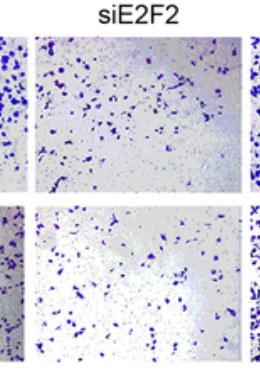

siNC+3-MA
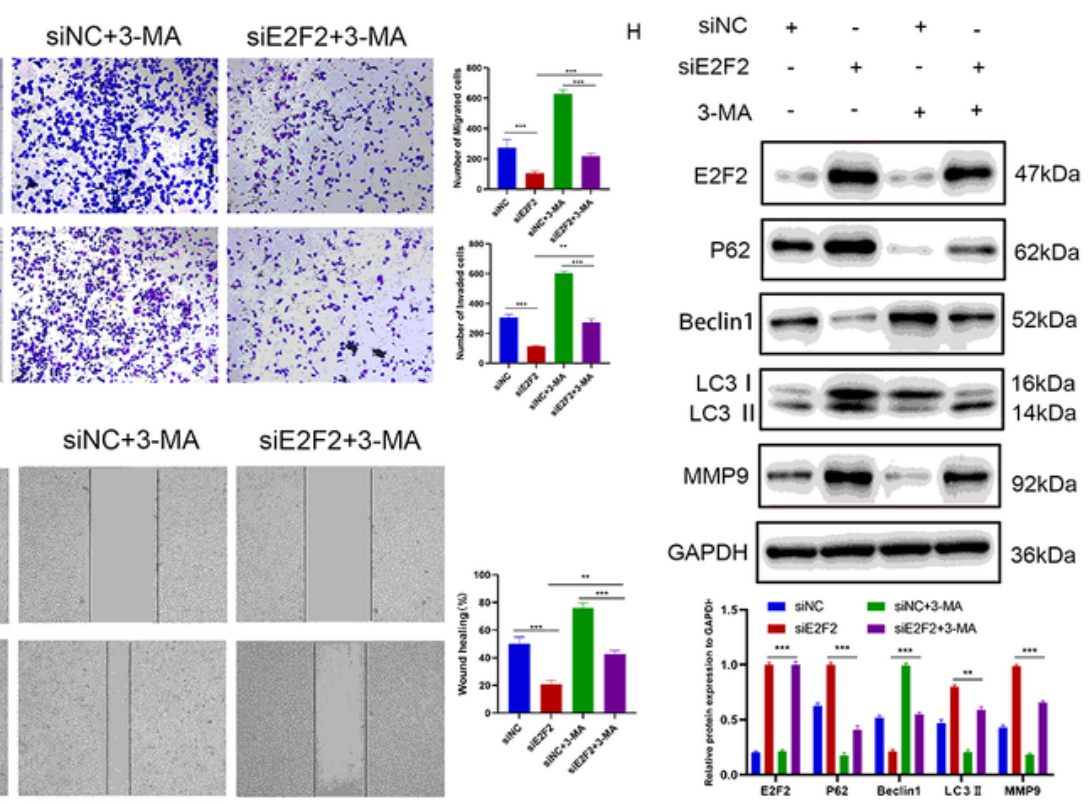

\section{Figure 7}

Effects of E2F2 expression levels on GC cell migration and invasion via autophagy mediation. (A) Western blotting analysis of P62, and LC3-II protein expression in GC cells transfected with GV141-Vector and GV141-E2F2. $\beta$-Actin was used as a loading control. (B) Western blotting analysis of P62, and LC3-II protein expression in GC cells transfected with siNC and siE2F2. $\beta$-Actin was used as a loading control. (C) Representative electron micrographs of autophagic vesicles in GC cells transfected with GV141-Vector 
and GV141-E2F2 and in GC cells transfected with siNC and siE2F2. (D) GC cells were transfected with GV141-Vector and GV141-E2F2 for $24 \mathrm{~h}$. Comparison of the migration and invasion GC cells using transwell compartments. (E) Wound-healing assay comparing the motility of GC cells. The wound-healing area was analyzed using the ImageJ software. (F) GC cells were transfected with siNC and siE2F2 or treated with phosphate-buffered saline (control) or 3-methyladenine $(2 \mathrm{mM})$ or a combination of both treatments for $24 \mathrm{~h}$. Comparison of the migration and invasion of GC cells using transwell compartments. (G) Wound-healing assay comparing the motility of GC cells. (h) Western blotting analysis of P62, Beclin1, LC3-II and MMP9 protein expression. $\beta$-Actin was used as a loading control. Data are presented

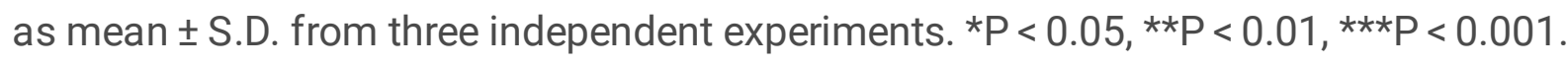


A
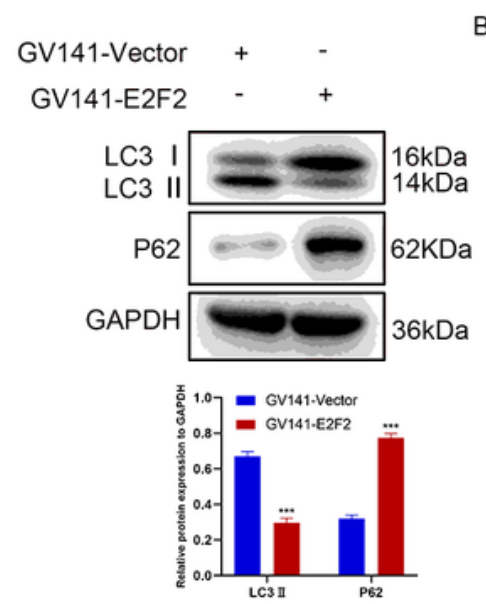

D

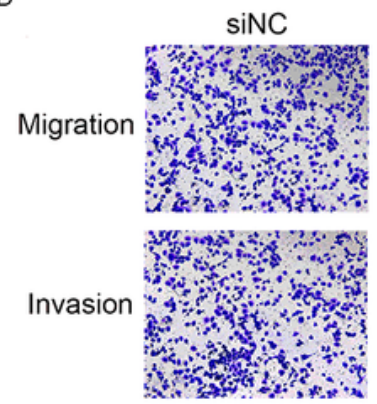

B
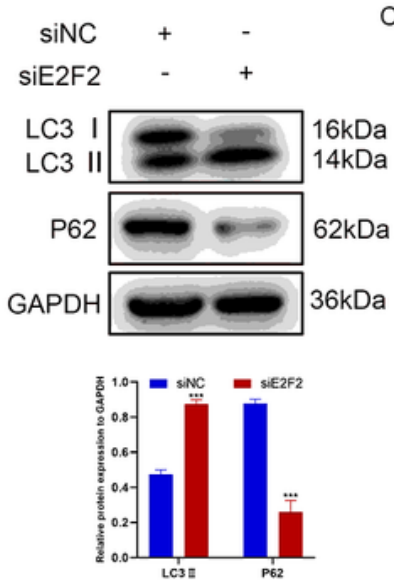

C
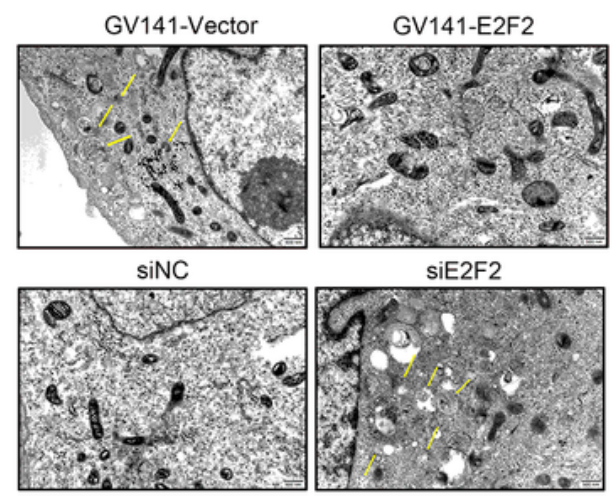

E

SiE2F2
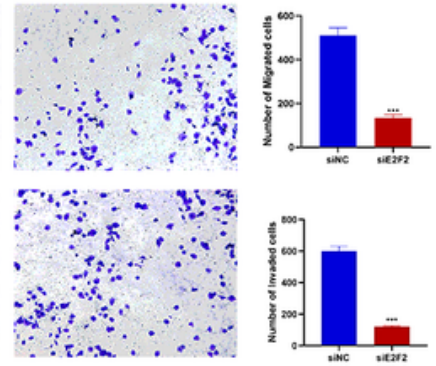

$24 h$

siNC

siE2F2

Oh
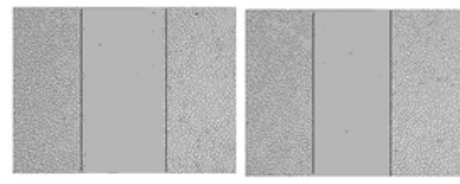

$\because 48$
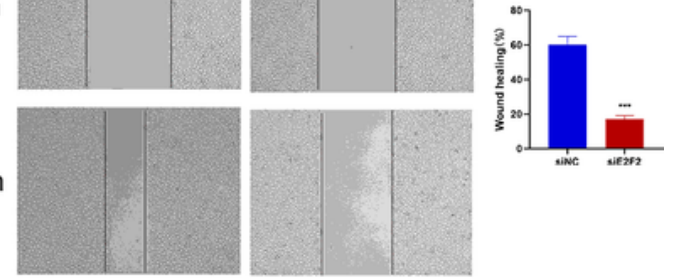

F

siNC

SiE2F2

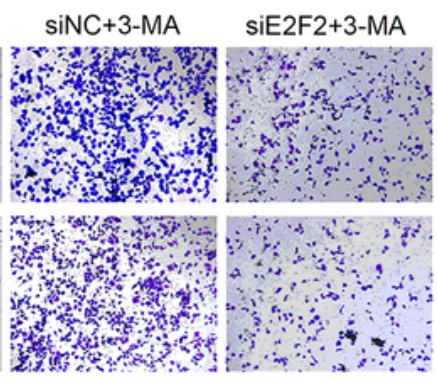

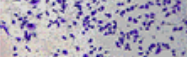

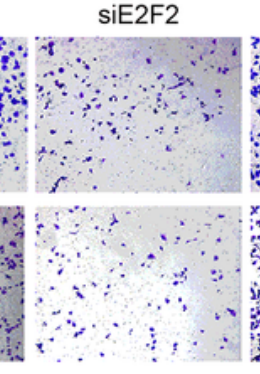

siNC+3-MA
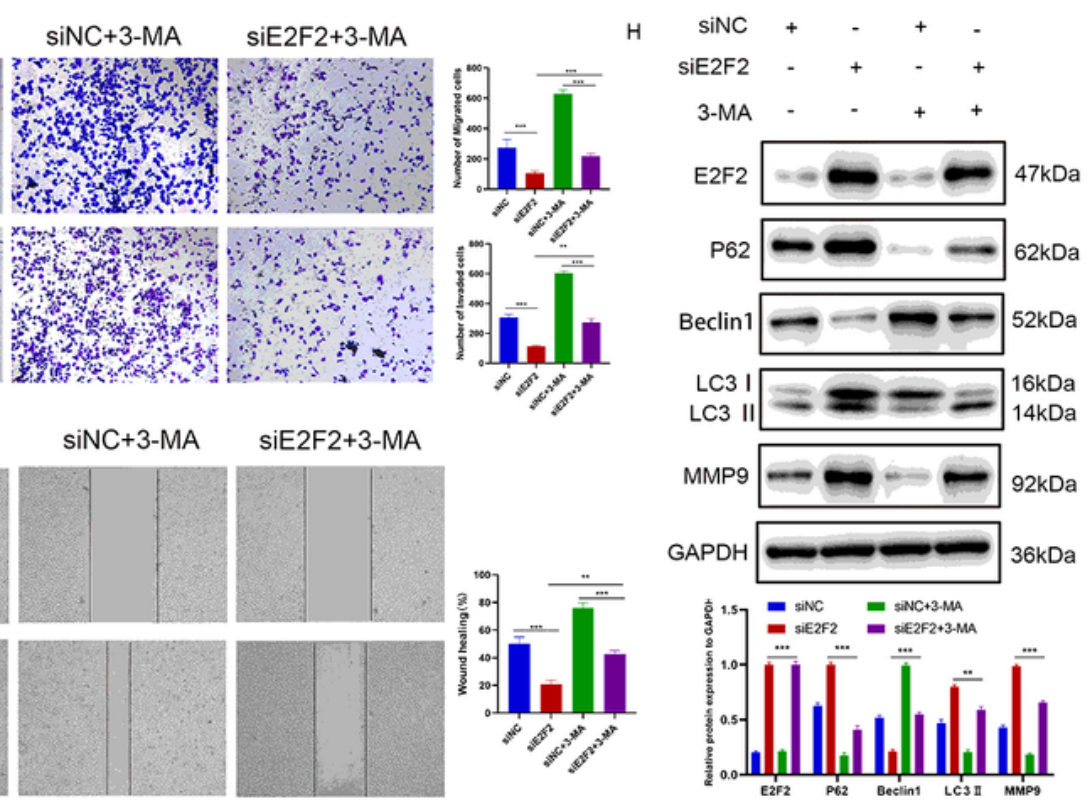

\section{Figure 7}

Effects of E2F2 expression levels on GC cell migration and invasion via autophagy mediation. (A) Western blotting analysis of P62, and LC3-II protein expression in GC cells transfected with GV141-Vector and GV141-E2F2. $\beta$-Actin was used as a loading control. (B) Western blotting analysis of P62, and LC3-II protein expression in GC cells transfected with siNC and siE2F2. $\beta$-Actin was used as a loading control. (C) Representative electron micrographs of autophagic vesicles in GC cells transfected with GV141-Vector 
and GV141-E2F2 and in GC cells transfected with siNC and siE2F2. (D) GC cells were transfected with GV141-Vector and GV141-E2F2 for $24 \mathrm{~h}$. Comparison of the migration and invasion GC cells using transwell compartments. (E) Wound-healing assay comparing the motility of GC cells. The wound-healing area was analyzed using the ImageJ software. (F) GC cells were transfected with siNC and siE2F2 or treated with phosphate-buffered saline (control) or 3-methyladenine (2 $\mathrm{mM})$ or a combination of both treatments for $24 \mathrm{~h}$. Comparison of the migration and invasion of GC cells using transwell compartments. (G) Wound-healing assay comparing the motility of GC cells. (h) Western blotting analysis of P62, Beclin1, LC3-II and MMP9 protein expression. $\beta$-Actin was used as a loading control. Data are presented

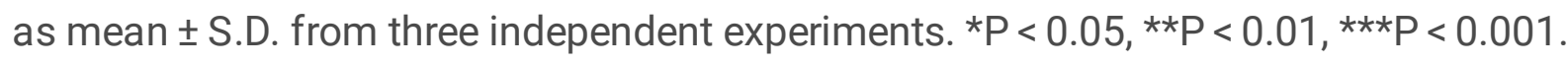

\section{Supplementary Files}

This is a list of supplementary files associated with this preprint. Click to download.

- SupplementaryTable1.doc

- SupplementaryTable1.doc 